\title{
Life-Cycle Portfolio Choice, the Wealth Distribution and Asset Prices*
}

\author{
Felix Kubler \\ Dept. of Banking and Finance \\ University of Zurich \\ and Swiss Finance Institute \\ fkubler@gmail.com
}

\author{
Karl Schmedders \\ Dept. of Business Administration \\ University of Zurich \\ and Swiss Finance Institute \\ karl.schmedders@business.uzh.ch
}

December 14, 2011

\begin{abstract}
In this paper we examine the volatility of asset returns in a canonical stochastic overlapping generations economy with sequentially complete markets. We show that movements in the intergenerational wealth distribution strongly affect asset prices since older generations have a lower propensity to save than younger generations. We investigate effects of aggregate shocks on the wealth distribution and show that they are generally small if agents have identical beliefs. Differences in opinion, however, can lead to large movements in the wealth distribution even when aggregate shocks are absent. The interplay of belief heterogeneity and life-cycle investments leads to considerable changes in the wealth distribution which, in turn, result in substantial asset price volatility. In fact, the model generates realistic second moments of asset returns.
\end{abstract}

Keywords: OLG economy, heterogeneous beliefs, life-cycle portfolio choice, wealth distribution, market volatility.

JEL Classification Codes: D53, E21, G11, G12.

${ }^{*}$ We thank seminar audiences at the University of Oxford, University of Frankfurt, University of Warwick, University of Zurich, Universitat Autonoma de Barcelona, Wuhan University, 2010 NSF/NBER/CEME conference on general equilibrium and mathematical economics at NYU, 2010 ICE summer workshop at the University of Chicago, 2010 SAET conference at Singapore, Institute for Advanced Studies and University of Vienna for comments and are grateful to Don Brown, Markus Brunnermeier, David Easley, Walt Pohl, Kevin Reffett, and Paolo Siconolfi for helpful discussions on the subject. We are indebted to the Swiss Finance Institute for financial support. 


\section{Introduction}

How does the distribution of wealth in an economy evolve over time and how do movements in the wealth distribution affect asset prices and the interest rate? To answer these important questions, we examine a canonical stochastic OLG model with dynamically complete markets. In the presence of uncertainty, asset prices depend both on the exogenous shock and the distribution of wealth (at the beginning of the period). If beliefs are identical then the wealth distribution changes little in equilibrium and the resulting impact on asset prices is quantitatively tiny. Differences in beliefs, however, lead agents to place large bets against each other and, as a result, wealth shifts across agents and across generations. Such changes in the wealth distribution strongly affect asset prices since older generations have a much higher propensity to consume than younger generations and as a result have much stronger incentives to divest of their asset investments. Put differently, prices of long-lived securities are typically considerably lower when 'old' generations hold most of the wealth than when 'young' generations hold most of the wealth in the economy. Belief heterogeneity leads to considerable changes in the wealth distribution which in turn result in substantial asset price volatility.

There is a large literature on the evolution of the wealth distribution and the effects of the wealth distribution on prices in general equilibrium models. In a model with infinitely lived agents, identical beliefs and complete financial markets there are no endogenous movements in the wealth distribution in equilibrium; all shocks are perfectly smoothed out and the wealth distribution as well as prices and choices just depend on the current exogenous shock (Judd et al., 2003). If beliefs differ, the wealth distribution changes in the short run, but in the long run only the agents with correct beliefs survive (see e.g. Sandroni, 2000, and Blume and Easley, 2006). When markets are incomplete these results are no longer true. However, under identical beliefs, in the stochastic growth model with ex ante identical agents and partially uninsurable income shocks, market incompleteness does not seem to matter quantitatively. Krusell and Smith (1998) show that in this model macroeconomic aggregates can be almost perfectly described using only the mean of the wealth distribution. Incomplete financial markets alone, therefore, cannot generate movements in asset prices as a result of (mean-preserving) movements in the wealth distribution.

In models with overlapping generations, the distribution of wealth across generations has potentially large effects on stock returns and the interest rate, since 'old' agents have a much higher marginal propensity to consume than 'young' agents. This fact was first discovered by Huffman (1987). He points out that a stochastic OLG model can "yield price volatility that would be difficult to rationalize within the context of other models." However, in many specifications of the model, the distribution of wealth moves little in response to aggregate shocks and has a minor effect on aggregate variables. Rios-Rull (1996) shows that the cyclical properties of a calibrated 
life-cycle model (with identical beliefs) are very similar to the properties of the model with a single infinitely lived agent. Storesletten et al. (2007) consider a model of an exchange economy with incomplete markets and identical beliefs. The fact that their computational strategy yields accurate results shows that just as in Krusell and Smith (1998), movements in the wealth distribution are negligible in their model. Models with demographic changes deliver different results. The wealth distribution moves due to changes in the size of different cohorts and these movements have strong effects on asset prices (see, e.g., Geanakoplos et al., 2004). ${ }^{1}$

The main result of this paper is that relatively small belief differences across agents in an OLG economy lead to large movements in the wealth distribution which, in turn, strongly impact aggregate variables. We examine a canonical stochastic OLG model with dynamically complete markets and assume that all agents have log-utility. Under this assumption there exists a recursive equilibrium with linear consumption policies and linear pricing functions. This feature enables us to analyze models with a large number of generations and substantial intra-generational heterogeneity. We begin our analysis by examining a stylized specification of our OLG model similar to the model in Huffman (1987). For this model we can derive closed-form solutions for the price of a stock ("Lucas tree") and the risk-free rate. The analytical solutions clearly demonstrate that the wealth distribution in the economy affects different assets differently. The tree price varies greatly with the wealth distribution while the risk-free rate is constant over an economically relevant (large) set of possible wealth distributions. The analytical results, therefore, suggest that our parsimonious OLG model can, via movements in the wealth distribution, simultaneously generate substantial stock price volatility and modest interest rate volatility.

We continue our theoretical analysis by proving two theorems contrasting OLG economies with identical beliefs and aggregate uncertainty with OLG economies with heterogeneous beliefs and no uncertainty in endowments and dividends. We first demonstrate that the OLG model with identical beliefs exhibits a stochastic steady state with a constant wealth distribution (conditional on exogenous shocks) if all endowments and dividends are collinear. Asset prices and consumption allocations only depend on the exogenous shock. This result insinuates that a parsimonious OLG model with identical beliefs and aggregate uncertainty cannot generate endogenous stock price volatility substantially exceeding exogenous dividend volatility for the simple reason of inadequate movements in the wealth distribution. Our result provides a theoretical explanation for the findings in Rios-Rull (1996) and Storesletten et al. (2007). For OLG economies with heterogeneous beliefs we can establish an opposing result. For any given stock return volatility and any arbitrarily small (positive) interest rate volatility, we construct an OLG economy in which the

\footnotetext{
${ }^{1}$ Benhabib et al. (2011) characterize the dependence of the wealth distribution in an OLG framework on technology, preferences and fiscal policy instruments. In particular, they examine wealth inequality and determine the extreme right tail of the wealth distribution. However, they do not analyze movements in the wealth distribution nor their impact on asset prices.
} 
(unique) equilibrium exhibits at least this stock return volatility and at most the given interest rate volatility. This result holds despite the fact that all agents agree on the distribution of the stock's dividends (it pays one unit of the consumption good in all states). The key idea behind the construction of the equilibrium is to choose beliefs in the Huffman-style OLG economy that lead to large movements in the wealth distribution which then yield the desired values for the price volatility of the assets.

Our theoretical results prompt the question whether they extend to realistically calibrated OLG economies. We first answer this question for OLG economies with identical beliefs. Agents' endowments in the economy are given by a life-cycle income function that was estimated from the Consumer Expenditure Survey (CEX) and the Panel Study of Income Dynamics (PSID). We deliberately choose shocks to endowments and dividends that are considerably larger than in the data so that the resulting models generate higher asset price volatility than properly calibrated models. Despite the large shocks, the resulting movements in the intergenerational wealth distribution are generally tiny. Not surprisingly, the stock return volatility is quite small and, in relation, the interest rate volatility is large. As Campbell (1999) points out, standard models cannot explain why the observed volatility of real US stock returns is so high in relation to the volatility of the short-term real interest rate. Our theoretical analysis and the numerical results show that this is also true for models with overlapping generations.

In the final step and most important step of the analysis, we examine the variability of the wealth distribution and the resulting asset price volatility for OLG economies with economically sensible belief differences. Agents' endowments are given by the afore-mentioned estimated lifecycle income process. Both endowments and dividends exhibit no uncertainty. If beliefs are identical in such an economy, then the unique long-run equilibrium is a steady state with a constant wealth distribution and constant asset prices. For economies with heterogeneous beliefs, however, the predictions of the model are dramatically different. We consider three different specifications for beliefs. There are three types of agents in the OLG economy. A common feature of all three specifications is that agents of type 1 always hold the correct beliefs. In the first specification, termed "persistent subjective beliefs", agents of types 2 and 3 have beliefs deviating antisymmetrically from the correct beliefs. Beliefs of agents of the same type are identical across generations. We vary both the proportion of type 1 agents as well as the magnitude of belief deviation for agents of the other two types. The stock return volatility in this economy exceeds the corresponding value of the homogeneous-beliefs model (with rather unrealistic aggregate shocks) for all examined beliefs deviations whenever the proportion of type 1 agents falls below 50 percent. In fact, for many parameter combinations the stock return volatility matches or exceeds the second moments observed in U.S. data. The large values for the stock return volatility are generally accompanied by very low values for the interest rate volatility. The wealth distribution in this economy exhibits very large movements. Moreover, it correlates with the stock price as predicted by our theoretical 
analysis. When the young are rich, the stock price tends to be high; when the old are rich, the stock price tends to be low.

An unattractive feature of our first belief specification is that agents do not learn. Type 2 and 3 agents do not revise their beliefs during their life cycle. While we need to remain silent on learning, introducing Bayesian learning in our framework renders the model intractable, we examine two modifications of the first specification. In the second belief specification, termed "converging beliefs", agents' beliefs converge to the correct beliefs as they age. That is, agents of types 2 and 3 always enter the economy with incorrect prior beliefs but 'learn' while they are alive. OLG models with this belief specification yield almost the same quantitative results as models with persistent subjective beliefs. As long as there is sufficient belief heterogeneity among the young there are large movements in the wealth distribution. In the third specification, termed "temporary disagreement", agents of types 2 and 3 typically have the correct beliefs but with low probability a regime shift occurs. After such a shift, type 2 and 3 agents have temporarily antisymmetric incorrect beliefs. For many parameter values, this belief specification also leads to high volatility.

Our model violates the common prior assumption that underlies much of applied general equilibrium modeling. As Morris (1995) points out, this assumption does not follow from rationality. However, any reasonable model that attempts to explain prices in financial markets needs to impose some discipline on the choice of beliefs. The focus of this paper is to highlight the large effects of small differences in beliefs, but we do not present a model which explains these differences. Kurz and Motolese (2001) use a theory of rational beliefs and argue in the context of an OLG economy with two-period-lived agents that belief heterogeneity is "the most important propagation mechanism of economic volatility." Our results support this finding but the underlying economic mechanism in our model with long-lived agents is quite different. In behavioral economics there are various models and explanations for different beliefs, see e.g. Bracha and Brown (2010).

Following Harrison and Kreps (1978), there is a large literature in finance that examines the effects of differences in beliefs and speculation on asset prices and bubbles (see, e.g., Scheinkman and Xiong, 2003). This literature has little relation to our paper; in our economy bubbles are impossible (see Santos and Woodford, 1997) and speculation in the sense of Harrison and Kreps (1978) is ruled out by the absence of short-sale constraints. There is also a large literature on the survival and price impact of noise traders, i.e. agents with wrong beliefs, see, among many others, DeLong et al. (1990), and Kogan et al. (2006). In our economy, new agents with wrong beliefs are born every period, so these have a persistent price impact. The relevant question for us is whether this price impact is quantitatively relevant.

The remainder of this paper is organized as follows. In Section 2 we describe the OLG model 
and introduce linear recursive equilibria. Section 3 illustrates the main mechanism in the context of special cases that allow for general theoretical statements. In Section 4 we discuss the effects of exogenous shocks on asset prices. Section 5 considers model specifications without uncertainty but with differences in beliefs. Section 6 concludes. The appendix contains all proofs and a description of the numerical method.

\section{Model}

In this section we first describe our model of stochastic overlapping generations economies. Subsequently we show that the unique equilibrium of our OLG model allows for a linear recursive formulation.

\subsection{Stochastic OLG economies}

Time is indexed by $t=0,1,2, \ldots$. A time-homogeneous Markov chain of exogenous shocks $\left(s_{t}\right)$ takes values in the finite set $\mathcal{S}=\{1, \ldots, S\}$. The $S \times S$ Markov transition matrix is denoted by $\Pi$. We represent the evolution of time and shocks in the economy by a countably infinite event tree $\Sigma$. The root node of the tree represents the initial shock $s_{0}$. Each node of the tree, $\sigma \in \Sigma$, describes a finite history of shocks $\sigma=s^{t}=\left(s_{0}, s_{1}, \ldots, s_{t}\right)$ and is also called date-event. We use the symbols $\sigma$ and $s^{t}$ interchangeably. To indicate that $s^{t^{\prime}}$ is a successor of $s^{t}$ (or $s^{t}$ itself) we write $s^{t^{\prime}} \succeq s^{t}$.

At each date-event $H$ agents commence their economic lives; they live for $N$ periods. An individual is identified by the date-event of his birth, $\sigma=s^{t}$, and his type, $h=1, \ldots, H$. The age of an individual is denoted by $a=1, \ldots, N$; he consumes and has endowments at all nodes $s^{t+a-1} \succeq s^{t}, a=1, \ldots, N$. An agent's individual endowments are a function of the shock and his age and type alone, i.e. $e^{s^{t}, h}\left(s^{t+a-1}\right)=e^{a, h}\left(s_{t+a-1}\right)$ for some functions $e^{a, h}: \mathcal{S} \rightarrow \mathbb{R}_{+}$, for all $h=1, \ldots, H, a=1, \ldots, N$.

Each agent has an intertemporal time-separable expected utility function,

$$
U^{s^{t}, h}(c)=\log \left(c\left(s^{t}\right)\right)+\sum_{a=1}^{N-1} \delta^{a} \sum_{s^{t+a} \succeq s^{t}} \pi^{a, h}\left(s^{t+a} \mid s^{t}\right) \log \left(c\left(s^{t+a}\right)\right) .
$$

The discount factor $\delta>0$ is constant and identical across agents, while the subjective probabilities $\pi^{a, h}\left(\sigma^{\prime} \mid \sigma\right)>0, \sigma^{\prime} \succeq \sigma$, may vary with age $a$ and type $h$. The Markov chain describing the agents' subjective beliefs ${ }^{2}$ may not be time-homogenous and vary with age. In particular it may differ from the "true" law of motion generated by $\Pi$.

\footnotetext{
${ }^{2}$ We denote the Markov transition matrix for an agent's subjective law of motion by $\pi^{a, h}$. That is, the agent who is currently of age $a$ assigns the probability $\pi^{a, h}\left(s, s^{\prime}\right)$ to a transition from the current exogenous state $s$ to the state $s^{\prime}$ in the next period when he is of age $a+1$. Occasionally it is necessary to refer to multi-step probabilities or to transition probabilities between nodes across the event tree. We denote such probabilities by $\pi^{a, h}\left(\sigma^{\prime} \mid \sigma\right)$ for nodes $\sigma^{\prime} \succeq \sigma$. The same convention applies to the "true" law of motion generated by $\Pi$.
} 
At each date-event $s^{t}$, there are $S$ Arrow securities in zero net supply available for trade. Prices of the Arrow securities are denoted by $q\left(s^{t}\right) \in \mathbb{R}^{S}$. The portfolio of such securities held by agent $(\sigma, h)$ is denoted by $\theta^{\sigma, h}\left(s^{t}\right) \in \mathbb{R}^{S}$. We use subscripts to indicate the Arrow security for a particular shock. The price at node $s^{t}$ of the Arrow security paying (one unit of the consumption good) at date-event $\left(s^{t}, s_{t+1}\right)$ is denoted by $q_{s_{t+1}}\left(s^{t}\right)$. Similarly, the holding of agent $(\sigma, h)$ of this security is denoted by $\theta_{s_{t+1}}^{\sigma, h}\left(s^{t}\right)$.

There is a Lucas tree in unit net supply paying dividends $d\left(s^{t}\right)>0$. Dividends are a function of the shock alone, so $d\left(s^{t}\right)=d\left(s_{t}\right)$ for some function $d: \mathcal{S} \rightarrow \mathbb{R}_{++}$. Let $\phi^{\sigma, h}\left(s^{t}\right)$ denote the holding of individual $(\sigma, h)$ at date-event $s^{t}$ and let $p\left(s^{t}\right)$ denote the price of the tree at that node.

Observe that the presence of a complete set of Arrow securities ensures that markets are dynamically complete. It is, therefore, without loss of generality that our economy has only a single Lucas tree since its primary purpose is to ensure that aggregate consumption exceeds aggregate endowments. The aggregate endowment in the economy is $\omega\left(s^{t}\right)=\omega\left(s_{t}\right)=d\left(s_{t}\right)+$ $\sum_{a=1}^{N} \sum_{h=1}^{H} e^{a, h}\left(s_{t}\right)$.

At time $t=0$, in addition to the $H$ new agents $\left(s_{0}, h\right), h=1, \ldots, H$, commencing their economic lives, there are individuals of each age $a=2, \ldots, N$ and each type $h=1, \ldots, H$ present in the economy. We denote these individuals by $\left(s^{1-a}, h\right)$ for $h=1, \ldots, H$ and $a=2, \ldots, N$. They have initial tree holdings $\phi^{s^{1-a}, h}$ summing up to 1 . These holdings determine the 'initial condition' of the economy.

\subsection{Sequential competitive equilibrium}

The consumption at date-event $s^{t}$ of the agent of type $h$ born at node $s^{t-a+1}$ is denoted $c^{s^{t-a+1}, h}\left(s^{t}\right)$. Whenever possible we write $c^{a, h}\left(s^{t}\right)$ instead. Similarly, we denote this agent's asset holdings by $\phi^{a, h}\left(s^{t}\right)$ and $\theta^{a, h}\left(s^{t}\right)$. This simplification of the notation allows us to use identical notation for the variables of individuals "born" at $t=0$ and later as well as those of individuals born prior to $t=0$.

A sequential competitive equilibrium is a collection of prices and choices of individuals

$$
\left(q\left(s^{t}\right), p\left(s^{t}\right),\left(\theta^{a, h}\left(s^{t}\right), \phi^{a, h}\left(s^{t}\right), c^{a, h}\left(s^{t}\right)\right)_{a=1, \ldots, N ; h=1, \ldots, H}\right)_{s^{t} \in \Sigma}
$$

such that markets clear and agents optimize.

(1) Market clearing equations:

$$
\sum_{a=1}^{N-1} \sum_{h=1}^{H} \phi^{a, h}\left(s^{t}\right)=1, \quad \sum_{a=1}^{N-1} \sum_{h=1}^{H} \theta^{a, h}\left(s^{t}\right)=0 \quad \text { for all } s^{t} \in \Sigma .
$$

(2) For each $s^{t}$, individual $\left(s^{t}, h\right), h=1, \ldots, H$, maximizes utility:

$$
\left(c^{s^{t}, h}, \phi^{s^{t}, h}, \theta^{s^{t}, h}\right) \in \arg \max _{c \geq 0, \phi, \theta} U^{s^{t}, h}(c) \quad \text { s.t. }
$$


budget constraint for $a=1$

$$
c\left(s^{t}\right)-e^{1, h}\left(s_{t}\right)+q\left(s^{t}\right) \cdot \theta\left(s^{t}\right)+p\left(s^{t}\right) \phi\left(s^{t}\right) \leq 0,
$$

budget constraints for all $s^{t+a-1} \succeq s^{t}, a=2, \ldots, N-1$

$$
c\left(s^{t+a-1}\right)-e^{a, h}\left(s_{t+a-1}\right)-\underbrace{\left(\theta_{s_{t+a-1}}\left(s^{t+a-2}\right)+\phi\left(s^{t+a-2}\right)\left(p\left(s^{t+a-1}\right)+d\left(s_{t+a-1}\right)\right)\right)}_{\text {beginning-of-period cash-at-hand }}+
$$

$$
\underbrace{\left(q\left(s^{t+a-1}\right) \cdot \theta\left(s^{t+a-1}\right)+p\left(s^{t+a-1}\right) \phi\left(s^{t+a-1}\right)\right)}_{\text {end-of-period investment }} \leq 0,
$$

budget constraint for all $s^{t+a-1} \succeq s^{t}, a=N$

$c\left(s^{t+N-1}\right)-e^{a, h}\left(s_{t+N-1}\right)-\left(\theta_{s_{t+N-1}}\left(s^{t+N-2}\right)+\phi\left(s^{t+N-2}\right)\left(p\left(s^{t+N-1}\right)+d\left(s_{t+N-1}\right)\right)\right) \leq 0$.

The utility maximization problems for the agents $\left(s^{1-a}, h\right), a=2, \ldots, N, h=1, \ldots, H$, who are born before $t=0$ are analogous to the optimization problems for agents $\left(s^{t}, h\right)$. The budget equation for agents of age $N$ shows that these agents do not invest anymore but instead consume their entire wealth. As a consequence their portfolios do not appear in the market-clearing equations.

The price of a riskless bond in this setting is simply equal to the sum of the prices of the Arrow securities. We denote the price of the riskless bond by $1 / R^{f}$, where $R^{f}$ denotes the risk-free rate.

\subsection{Linear recursive equilibria}

Huffman (1987) considers an OLG economy with incomplete markets, a single Lucas-tree, and logarithmic utility in which agents receive an individual endowment only in the first period of their life. These assumptions lead to a closed-form function for the price of the tree. In our OLG model such a closed-form pricing function does not exist. But the assumption of logarithmic utility allows us to express the equilibrium consumption allocations, the price of the Lucas-tree, and the riskless rate as simple functions of state variables. The natural endogenous state variables in the OLG economy are the beginning-of-period cash-at-hand positions of the agents of ages $a=$ $2, \ldots, N-1$. Cash-at-hand of agents of age $N$ who are in the last period of their economic lives do not need to be included in the state space. Agents of age $a=1$ always enter the economy without any initial cash-at-hand. Let $\kappa^{a, h}\left(s^{t}\right)$ denote beginning-of-period cash-at-hand of an individual of age $a$ and type $h$ at node $s^{t}$, that is,

$$
\kappa^{a, h}\left(s^{t}\right)=\phi^{a-1, h}\left(s^{t-1}\right)\left(p\left(s^{t}\right)+d\left(s^{t}\right)\right)+\theta_{s_{t}}^{a-1, h}\left(s^{t-1}\right)
$$

for $a=2, \ldots, N-1$ and $h=1, \ldots, H$. The following theorem is proved in the appendix.

TheOREM 1 Given a shock $s_{t}=s \in \mathcal{S}$, consumption of the agent of age $a=1, \ldots, N-1$, and type 
$h=1, \ldots, H$, is a linear function of the individual cash-at-hand positions, that is

$$
c^{a, h}\left(s^{t}\right)=\alpha_{1 s}^{a, h}+\sum_{j=2}^{N-1} \sum_{i=1}^{H} \alpha_{j i s}^{a, h} \kappa^{j, i}\left(s^{t}\right),
$$

for some coefficients $\alpha_{j i s}^{a, h} \geq 0$. The price of the tree is also a linear function of the individual cash-at-hand positions, that is

$$
p\left(s^{t}\right)=\beta_{1 s}+\sum_{a=2}^{N-1} \sum_{h=1}^{H} \beta_{a h s} \kappa^{a, h}\left(s^{t}\right),
$$

for some coefficients $\beta_{\text {ahs }} \geq 0$. The riskless rate $R^{f}$ satisfies the relation

$$
1 / R^{f}\left(s^{t}\right)=\gamma_{1 s}+\sum_{a=2}^{N-1} \sum_{h=1}^{H} \gamma_{a h s} \kappa^{a, h}\left(s^{t}\right),
$$

for some coefficients $\gamma_{a h s} \geq 0$.

The three linear functions in the theorem look deceivingly simple. Observe that an agent's cash-at-hand $\kappa^{a, h}\left(s^{t}\right)$ depends on the price of the Lucas-tree $p\left(s^{t}\right)$ whenever he holds a nonzero position of the tree. Equation (2), therefore, is a fixed-point equation instead of a closed-form expression such as the pricing formula in Huffman (1987). Nevertheless the three formulas prove to be very helpful for our analysis because they enable us to compute the OLG equilibrium and to simulate the economy. Unfortunately, we cannot determine the coefficients $\alpha, \beta$, and $\gamma$ analytically unless we make additional assumptions, see Section 3.1 below. We describe how we can compute these quantities numerically in Appendix B.

The state of the economy comprises the exogenous shock $s \in \mathcal{S}$ and the endogenous vector of beginning-of-period cash-at-hand holdings $\kappa \equiv\left(\kappa^{a, h}\right)_{h=1, \ldots, H ; a=2, \ldots, N-1}$. A recursive equilibrium (for a general treatment of recursive equilibria in stochastic OLG economies see Citanna and Siconolfi, 2010) consists of a policy function that maps the state of the economy, $(s, \kappa)$, to current prices and choices as well as a transition function that maps the state in the current period to a probability distribution over states in the subsequent period.

An interesting special case arises in the absence of exogenous shocks. In this case, the dynamics of the wealth distribution depends crucially on agents' beliefs. The policy functions, however, are independent of beliefs.

Proposition 1 For given deterministic endowments and dividends, the coefficients $\alpha$ of the consumption functions (1) and the coefficients $\beta$ and $\gamma$ of the pricing functions (2) and (3) in Theorem 1 are independent of the specification of beliefs. That is, for given endowments and dividends, the consumption function is

$$
c^{a, h}\left(s^{t}\right)=\alpha_{1}^{a, h}+\sum_{j=2}^{N-1} \sum_{i=1}^{H} \alpha_{j i}^{a, h} \kappa^{j, i}\left(s^{t}\right),
$$


for some coefficients $\alpha_{j i}^{a, h}, a=1, \ldots, N-1, h=1, \ldots, H$, which do not depend on beliefs. The price of the Lucas-tree at any date event $s^{t}$ is given by an expression of the form

$$
p\left(s^{t}\right)=\beta_{1}+\sum_{a=2}^{N-1} \beta_{a} \sum_{h=1}^{H} \kappa^{a, h}\left(s^{t}\right)
$$

for some coefficients $\beta_{a}, a=1, \ldots, N-1$, which do not depend on beliefs. Similarly, the risk-free rate $R^{f}$ satisfies the relation

$$
1 / R^{f}\left(s^{t}\right)=\gamma_{1}+\sum_{a=2}^{N-1} \gamma_{a} \sum_{h=1}^{H} \kappa^{a, h}\left(s^{t}\right)
$$

for some coefficients $\gamma_{a}, a=1, \ldots, N-1$, which do not depend on beliefs.

Clearly the proposition does not generalize to economies with uncertain dividends. In such economies the beliefs of the agents owning the Lucas-tree matter for its price.

\section{Some theoretical results}

We first consider some stylized specifications of our general model for which we can prove analytical results about equilibrium asset prices and the wealth distribution.

\subsection{The intergenerational wealth distribution and asset prices}

We first examine a deterministic special case of our OLG model which admits an analytical solution. We assume that agents only have positive endowments in the first period of their lives. For notational simplicity, we consider the case $H=1$ since intragenerational heterogeneity adds little to the results in this section. This allows us to drop the superscript for the type throughout this section. We assume that $e^{a, 1}=e^{a}=0$, for $a=2, \ldots, N$ and that $e^{1}=1$. The Lucas-tree pays deterministic dividends $d>0$. The assumption of a deterministic economy allows us to assume without loss of generality that agents only trade in the stock, i.e., the endogenous state can be written as

$$
\kappa^{a,}(t)=\phi^{a-1}(t-1)(p(t)+d(t))
$$

for $a=2, \ldots, N-1$. The results in this section provide an important benchmark for our analysis below where we introduce uncertainty and heterogenous beliefs. Proposition 1 implies that the pricing and consumption functions in a model where all endowments and all dividends are constant across shocks (i.e., where shocks only play a role because agents can gamble on them) are the same as in the deterministic model.

The model without uncertainty is a special instance of the asset-pricing model in Huffman (1987). He also assumes that agents only receive endowments in the first period in their lives and that the only asset available for trade is the tree. While he allows for uncertainty, his result obviously also holds in a deterministic model. Huffman's (1987, p. 142) analysis yields the following 
coefficients for the linear tree price expression,

$$
\beta_{1}=\frac{\delta-\delta^{N}}{1-\delta^{N}}, \quad \beta_{a}=\frac{\delta-\delta^{N-a+1}}{1-\delta^{N-a+1}}, \quad \text { for } a=2, \ldots, N-1,
$$

for $\delta \neq 1$. Applying L'Hospital's rule as $\delta \rightarrow 1$ we obtain for $\delta=1$ the coefficients

$$
\beta_{1}=\frac{N-1}{N}, \quad \beta_{a}=\frac{N-a}{N-a+1}, \quad \text { for } a=2, \ldots, N-1 .
$$

All coefficients are positive and bounded above by 1 .

While Huffman considered an economy with a single tree, in our deterministic economy we can also easily determine the bond prices. While this price follows by the absence of arbitrage it is easier to derive using the Arrow-Debreu equilibrium as in the following proposition.

Proposition 2 In the deterministic economy with $e^{a}=0$, for $a=2, \ldots, N$, and $e^{1}=1$, the bondpricing coefficients $\gamma$ are

$$
\gamma_{1}=\frac{\delta}{(1+d) \sum_{j=0}^{N-1} \delta^{j}-1} \quad \text { and } \gamma_{a}=\frac{\sum_{j=1}^{N} \delta^{j}}{\left((1+d) \sum_{j=0}^{N-1} \delta^{j}-1\right) \sum_{j=0}^{N-a} \delta^{j}}, \quad a=2, \ldots, N-1 .
$$

Given the pricing functions for the bond and the tree, we can now ask how asset prices change with the wealth distribution. For the discussion of the benchmark model, we hypothetically assume an exogenously given wealth distribution. We consider the special case $\delta=1$. This assumption greatly simplifies the formulas. By continuity our qualitative insights carry over to economies with discount factors close to but different from 1.

For $\delta=1$, Equation (5) can be used to solve for the price of the tree and implies that the tree price must be

$$
p\left(s^{t}\right)=\frac{\frac{N-1}{N}+d\left(\sum_{a=2}^{N-1} \frac{N-a}{N-a+1} \phi^{a-1}\left(s^{t}\right)\right)}{1-\sum_{a=2}^{N-1} \frac{N-a}{N-a+1} \phi^{a-1}\left(s^{t}\right)} .
$$

Suppose the entire tree is held by the agents of a particular age $a \in\{2,3, \ldots, N-1\}$. (This cannot happen in equilibrium due to the zero endowment after the first period. However, the argument is also correct but more tedious for a holding of $1-\varepsilon$.) Then the tree price is

$$
p\left(s^{t}\right)=(N-a)(1+d)+\frac{a-1}{N} .
$$

If the entire tree is held by agents of age $N$ then the price is $p\left(s^{t}\right)=\beta_{1}=\frac{N-1}{N}$.

Since $\partial p\left(s^{t}\right) / \partial a<0$ we observe that the younger the agents holding the entire tree are the larger is its price. For agents of fixed age $a<N$ holding the tree and increasing values of $N$, the tree price grows without bound. If, on the contrary, the agents of age $N$ hold the entire tree, then its price is equal to $\frac{N-1}{N}$ and thus bounded above by 1 . So, the price of the Lucas-tree may vary greatly as the wealth distribution changes. 
In Appendix A we derive the price of the riskless bond from Equation (6),

$$
1 / R^{f}\left(s^{t}\right)=\frac{1}{N(d+1)-1}+\frac{\sum_{a=2}^{N-1}\left(\frac{1}{N-a+1}\right) \phi^{a-1}\left(s^{t}\right)}{1-\sum_{a=2}^{N-1}\left(1-\frac{1}{N-a+1}\right) \phi^{a-1}\left(s^{t}\right)} .
$$

If the agents of age $N$ have zero holdings of the tree then $\sum_{a=2}^{N-1} \phi^{a-1}\left(s^{t}\right)=1$ and the price of the riskless bond is constant,

$$
1 / R^{f}\left(s^{t}\right)=1+\frac{1}{N(d+1)-1} .
$$

If the entire tree is held by agents of age $N$ then the price of the riskless bond is $1 / R^{f}\left(s^{t}\right)=$ $\frac{1}{N(d+1)-1}$.

Observe that as long as the agents of age $N$ have zero tree holdings the risk-free rate is constant. This fact is perhaps somewhat surprising since the tree price may vary from large values such as $(N-2)(d+1)+\frac{1}{N}$ (if agents of age 2 hold the entire tree) to small values such as $(d+1)+\frac{N-2}{N}$ (if agents of age $N-1$ hold the entire tree). For large ranges of the wealth distribution there in no direct link between the risk-free rate and the price of the Lucas tree. In a deterministic economy, if agents of age $a$ hold the entire tree in the current period, agents of age $a+1$ will hold almost the entire tree in the next period. As a result, the price of the tree will slightly drop and the (deterministic) return of the tree will be small (possibly negative). In the described model specification, this absolute tree price decrease is independent of $a$, i.e., by the absence of arbitrage the interest rate must remain the same as the wealth is held by agents of ages 1 through $N-1$.

To illustrate the possible variability in asset prices, Table 1 displays the prices of the Lucas-tree and the riskless bond for an economy in which agents live for $N=240$ periods. The safe dividend of the tree is $d=1$. The tree price varies between 2.9917 and 476.00 without changes in the risk-free

\begin{tabular}{|c||c|c|c|c|c|c|c||c|}
\hline$a$ & 2 & 5 & 10 & 100 & 200 & 230 & 239 & 240 \\
\hline$p\left(s^{t}\right)$ & 476.00 & 470.02 & 460.04 & 280.41 & 80.829 & 20.954 & 2.9917 & 0.99583 \\
\hline $1 / R^{f}\left(s^{t}\right)$ & \multicolumn{10}{|c||}{1.0021} & 0.0020877 \\
\hline
\end{tabular}

Table 1: Prices $p\left(s^{t}\right)$ and $1 / R^{f}\left(s^{t}\right)$ if agents of age $a$ hold the entire Lucas-tree

rate.

The described price movements in the deterministic economy can only arise if we consider unanticipated shocks to the wealth distribution and even then they are only transitory. The wealth distribution converges quickly to a steady state distribution from any initial condition. Similarly, the tree price and the risk-free rate converge fast to their respective steady-state values. Nevertheless the observed effects prove to be important in our model. In an economy with heterogeneous beliefs the wealth distribution varies endogenously and no steady state exists. As a result, large price movements persist indefinitely. 


\subsection{The effect of aggregate shocks on the wealth distribution and prices}

Before turning to the analysis of heterogenous beliefs, we first state a benchmark result for economies with identical beliefs and aggregate shocks. The next theorem describes two benchmark specifications of our OLG model with aggregate uncertainty for which the wealth distribution remains constant along the equilibrium path and thus does not matter for equilibrium allocations and prices. While we can prove the theorem only for a specific initial condition, we found in many simulations that, if the economy starts from other initial conditions, then the equilibrium quickly converges to the stochastic steady state with a constant wealth distribution.

Theorem 2 Consider an economy where all agents $a=1, \ldots, N, h=1, \ldots, H$, have identical and correct beliefs, $\pi^{a, h}=\Pi$. Then, under either of the following two assumptions, there exist initial conditions $\kappa$ such that in the resulting equilibrium, prices and consumption choices are time invariant functions of the exogenous shock alone.

1. All endowments and dividends are collinear, i.e. for all agents $a=1, \ldots, N, h=1, \ldots, H$, it holds that

$$
\frac{e^{a, h}(s)}{e^{a, h}\left(s^{\prime}\right)}=\frac{d(s)}{d\left(s^{\prime}\right)} \quad \text { for all } s, s^{\prime}=1, \ldots, S .
$$

2. Shocks are i.i.d., i.e. for all shocks $s^{\prime}, \Pi\left(s, s^{\prime}\right)$ is independent of $s$, and endowments of all agents of age $a=1$ are collinear to aggregate endowments, i.e. for all $h=1, \ldots, H$,

$$
\frac{e^{1, h}(s)}{e^{1, h}\left(s^{\prime}\right)}=\frac{\omega(s)}{\omega\left(s^{\prime}\right)} \quad \text { for all } s, s^{\prime}
$$

See Appendix A for a proof of the theorem. Commonly applied realistic calibrations of asset pricing models deviate from the assumptions of Theorem 2 in at least two directions. Either labor endowments are assumed to be safe or shocks to labor endowments are assumed to be independent of shocks to dividends. The question arises whether such calibrations of our OLG model lead to substantially different equilibrium predictions. We investigate this question in Section 4 below.

\subsection{Differences in beliefs and asset price volatility}

To isolate the effects of beliefs, models with deterministic dividends and endowments serve as a useful benchmark for our analysis. For the discussion in this section we assume that $e^{a, h}(s)=e^{a, h}$ and $d(s)=d$ for all shocks $s \in \mathcal{S}$. By continuity, the results for such models are similar to those for models with very small shocks to these fundamentals. Thus we view this specification of the general model as a limiting case for economies with little uncertainty.

If in such a model agents have identical beliefs then it is equivalent to a deterministic OLG economy. The economy has a unique steady state, which is independent of beliefs, and for all initial conditions the unique equilibrium converges to this steady state. If agents have differences 
in beliefs, however, then a steady state does not exist and the wealth distribution changes along the equilibrium path. These changes can have very strong effects on asset prices as we show below.

For comparison, note that in a model with infinitely-lived agents and deterministic endowments and dividends, differences in beliefs do not affect asset prices (as long as all agents have identical time preferences). Although the wealth distribution may change over time and across shocks, all agents agree that the price of the tree should equal the discounted sum of its (safe) dividends.

If we impose no restrictions on beliefs we can obtain arbitrary movements in the wealth distribution across agents. We can construct beliefs such that in equilibrium, as $N$ or $\delta$ become large, the volatility of the tree price becomes arbitrarily large while the volatility of the bond price remains arbitrarily low. The following theorem states these facts formally.

THEOREm 3 Given any tree-return volatility, $\bar{v}<\infty$, and any bond-price volatility, $\underline{v}>0$, for any time horizon $T>1$ and any initial condition $\kappa \gg 0$, we can construct an economy where the stock return volatility is at least $\bar{v}$ while the interest rate volatility is at most $\underline{v}$, that is,

$$
\operatorname{Std}\left(R^{e}\right) \geq \bar{v}, \quad \operatorname{Std}\left(R^{f}\right) \leq \underline{v} .
$$

The proof in the appendix constructs economies with $\delta=1$, letting $N$ become arbitrarily large. In light of the benchmark case above, we can either hold $N$ fixed and choose $\delta$ and $\left(\pi^{a}\right)_{a=1, \ldots, N-1}$ or we can hold $\delta \geq 1$ fixed and choose $N$ and $\left(\pi^{a}\right)_{a=1, \ldots, N-1}$ in order to obtain the desired return volatility.

In an OLG model, movements in the wealth distribution can lead to large changes in the prices of long-lived assets without changing the short-term interest rate. The intuition above applies here, too. All agents believe that, with high probability, wealth will be passed down from agents of age $n$ to agents of age $n+1$, hence the bond-price stays relatively constant, independently of $n$ as long as it is smaller than $N$. The result accentuates that differences in beliefs can have potentially huge effects on the price of the long-lived asset in this economy. If we can freely choose beliefs over the exogenous shocks then we can generate arbitrary price volatility. The proof of the theorem shows that the price of the tree can move arbitrarily far away from the discounted present value of its dividends if these are discounted using the current interest rate. Following Harrison and Kreps (1978) there is now a large literature in finance that demonstrates how asset pricing bubbles can arise from differences in beliefs and speculation. It is important to note that in our model there can never be bubbles in equilibrium, see Santos and Woodford (1999). Nevertheless, the economy exhibits large swings in the price of the tree which could not be distinguished from an asset pricing bubble if we only examined prices and observed aggregate variables.

Constantinides and Duffie (1996) describe an economy that theoretically generates a much wider range of asset price processes than our OLG economy with heterogeneous beliefs. In their 
economy, agents have permanent idiosyncratic income shocks, agents' income risks are uninsurable, and there is a no-trade equilibrium. Moreover, any stochastic discount factor and so any arbitrage-free asset price process can be generated in equilibrium for appropriately chosen income processes. However, there is mixed evidence in the literature about the potential of their mechanism to be important in realistically calibrated models (see, e.g., Storesletten et al., 2007).

Similarly our theoretical result of Theorem 3 relies on a careful construction of heterogeneous beliefs for all agents. Thus, it gives no indication on the quantitative importance of the asset price volatility when beliefs exhibit small differences. We report equilibrium quantities for our OLG economy with heterogeneous beliefs in Section 5 below. Before we start with the numerical analysis of our mode, it is interesting to note that equilibrium price volatility in this economy relies crucially on the existence of a rich asset structure.

\subsection{Incomplete vs. complete markets}

In an OLG economy with a single tree but no other securities the pricing formula for the tree remains the same as in our OLG model. As the analysis in Huffman (1987) shows, there is a steady state with no trade even if beliefs are heterogeneous. In Huffman's economy, agents' consumption and savings decisions are independent of their beliefs, they depend only on the discount factor $\delta$ and the age of an agent. An agent of age $a$ always consumes a fixed fraction of his cash-at-hand, no matter what his expectations are for future prices. Therefore, in the absence of Arrow securities there is no complex trading in this economy and zero price volatility in equilibrium in the long run - for any beliefs and discount factors. On the contrary, when there is a complete set of Arrow Securities available for trade as in our OLG model, price volatility can be arbitrary. In this sense, a rich set of financial assets can lead to a huge increase in the volatility of the price of the tree.

\section{Aggregate uncertainty and identical beliefs}

Previous research revealed that in many specifications of the overlapping generations model with aggregate uncertainty the wealth distribution changes very little in equilibrium if beliefs are identical, see, for example, Rios-Rull (1996) and Storesletten et al. (2007). We replicate their findings in our model. The results serve as a useful benchmark for our analysis of OLG economies with heterogenous beliefs.

\subsection{A (rough) calibration}

We consider a specification of the model with calibrated labor income. A time period is meant to represent a quarter and so we assume that agents live for $N=240$ periods. We use the parameter values estimated by Davis et al. (2006) for a realistic calibration of life-cycle income. They follow 
the estimation strategy of Gourinchas and Parker (2002) and fit a 5th order polynomial to match average income from the Consumer Expenditure Survey (CEX) and the Panel Study of Income Dynamics (PSID). The resulting age-income profile is given by

$$
\begin{gathered}
\log \left(e^{a}\right)=6.62362+0.334901\left(\frac{a}{4}+20\right)-0.0148947\left(\frac{a}{4}+20\right)^{2}+3.63424 \cdot 10^{-4}\left(\frac{a}{4}+20\right)^{3} \\
-4.41169 \cdot 10^{-6}\left(\frac{a}{4}+20\right)^{4}+2.05692 \cdot 10^{-8}\left(\frac{a}{4}+20\right)^{5}
\end{gathered}
$$

for $a \leq 4 \cdot 43=172$ and $e^{a}=\frac{e^{172}}{2}$ for $a=173, \ldots, 240$. This profile is hump-shaped with a replacement rate at retirement of 50 percent.

We normalize aggregate endowments to be on average $\bar{\omega}=1$ and assume that the stock's average dividends are $\bar{d}=0.15$, i.e. labor endowments are normalized to add up to 0.85 on average. Assuming that dividends are 15 percent of aggregate endowments is motivated by the idea that the tree in this model represents both the aggregate stock market and some fraction of the housing market. The actual share of dividends in aggregate consumption is around 5 percent. The effects on volatility become larger as the dividend share becomes smaller. Fifteen percent certainly appears to be an adequate upper bound.

In this section we consider an economy with both endowment and dividend shocks - we abstract from idiosyncratic shocks because financial markets are complete - therefore there is only one type of agent per generation, so $H=1$. To make the point that aggregate shocks do not move the wealth distribution in this model, we deliberately consider rather large shocks; for smaller shocks, the resulting volatility effects are obviously much smaller. Specifically, let dividends and endowments be

$$
\begin{gathered}
d(1)=d(2)=0.15(1+\eta), d(3)=d(4)=0.15(1-\eta), \\
e^{a}(1)=e^{a}(3)=0.99 e^{a}, e^{a}(2)=e^{a}(4)=1.01 e^{a},
\end{gathered}
$$

respectively. We vary the magnitude $\eta$ of the dividend shock between 0.05 and 0.15 . In the data, quarterly dividends and aggregate consumption are essentially uncorrelated. In (detrended) levels both shocks to dividends and shocks to labor income are persistent. We choose the probability to remain in the same dividend state to be $2 / 3$ while the probability to stay in the same laborincome state is $3 / 4$. The standard deviation of labor income shocks is chosen to roughly match the data and we vary the size of the dividend shock in order to demonstrate that the magnitude of this shock affects asset price volatility but does not affect the wealth distribution.

A proper calibration of the model leads to a discount factor of $\delta>1$. As we observe in our analysis of heterogenous beliefs in the next section, the effects of belief heterogeneity on the volatility of the stock prices increase with $\delta$. Therefore, we deliberately choose $\delta=1$ to stack the deck against heterogenous beliefs. The resulting interest rate in the model is then slightly too high in comparison to the average real interest rate of annually 1 percent observed in the data (see Campbell, 1999). 


\subsection{Results}

Lettau and Uhlig (2002) report that the quarterly standard deviation of returns of S\&P-500 stocks in post-war US data is about 7.5 percent. On the other hand, the standard deviation of the quarterly real interest rate is around 1.4 percent; and as Campbell (1999) points out, a lot of this variation is due to inflation risk.

Table 2 reports the volatility of the tree returns and the real interest rate. The figures shows

\begin{tabular}{|c|c|c|c|}
\hline & $\eta=0.05$ & $\eta=0.1$ & $\eta=0.15$ \\
\hline $\operatorname{Std}\left(R^{f}\right)$ & 0.66 & 1.09 & 1.57 \\
\hline $\operatorname{Std}\left(R^{e}\right)$ & 1.23 & 2.00 & 2.83 \\
\hline
\end{tabular}

Table 2: Second moments (in \%) - aggregate shocks

that even for very large shocks to dividends, the standard deviation of tree returns remains much below the empirical value. Moreover, the figures point to the well-known close link between the stock-return volatility and the interest rate volatility in consumption-based asset pricing models with identical beliefs. This counterfactual result is caused by the lack of movements in the wealth distribution. To a first approximation, individual consumption only depends on the current shock and hence an agent's intertemporal Euler equation necessarily gives a close link between stock returns and the interest rate.

To illustrate the fact that the wealth distribution remains almost constant over time, we aggregate all agents' shares of beginning-of-period cash-at-hand $\kappa^{a, h}\left(s^{t}\right)$ into ten groups. The cash-athand shares of groups $1,2, \ldots, 10$ are

$$
\frac{\sum_{h=1}^{H} \sum_{a=1}^{24} \kappa^{a, h}\left(s^{t}\right)}{p\left(s^{t}\right)+d\left(s^{t}\right)}, \frac{\sum_{h=1}^{H} \sum_{a=25}^{48} \kappa^{a, h}\left(s^{t}\right)}{p\left(s^{t}\right)+d\left(s^{t}\right)}, \ldots, \frac{\sum_{h=1}^{H} \sum_{a=217}^{240} \kappa^{a, h}\left(s^{t}\right)}{p\left(s^{t}\right)+d\left(s^{t}\right)},
$$

respectively. Group 1 are the 72 agents who are in one of the first 24 periods of their lives, group 2 are the subsequent 72 agents who are in the 25 th to 48 th period of their lives, and so on. The larger the group number the older are the agents in the group.

We report results from a simulation over $100^{\prime} 000$ periods of the economy with $\eta=0.15$. Table 3 displays the average cash-at-hand shares of all ten groups as well as the corresponding standard deviations. The standard deviations vary around $7 \times 10^{-5}$, thus we report the values in $1 / 1000$ of a percent. The figures in the table clearly show that the wealth distribution practically does not move in this calibration. Not surprisingly, we obtain similar results for smaller values of the dividend shock $\eta$.

In sum, the results for our roughly calibrated OLG economy confirm some well-known failures of parsimonious asset pricing models. The observed volatility of stock returns is considerably higher than the observed dividend volatility. For realistic parameter values, parsimonious models 


\begin{tabular}{|l|rrrrr|}
\hline Group & 1 & 2 & 3 & 4 & 5 \\
\hline average (\%) & 0.5 & 2.0 & 4.4 & 7.6 & 11.5 \\
\hline std. dev. $\left(\frac{1}{1000} \%\right)$ & 7.4 & 7.9 & 7.1 & 5.4 & 2.8 \\
\hline \hline Group & 6 & 7 & 8 & 9 & 10 \\
\hline average (\%) & 15.6 & 19.1 & 19.5 & 14.1 & 5.7 \\
\hline std. dev. $\left(\frac{1}{1000} \%\right)$ & 2.7 & 6.3 & 13.4 & 8.4 & 1.1 \\
\hline
\end{tabular}

Table 3: Wealth distribution - aggregate shock $\eta=0.15$

cannot match the observed high return volatility. And for the often unrealistic parameter values that do allow these models to deliver a larger return volatility, the accompanying interest rate volatility grows, too, and is much larger than in the data. This excess return volatility puzzle is one of many (related) asset pricing puzzles, such as, among others, the equity premium puzzle and the Sharpe ratio puzzle, see Campbell (1999).

\section{Different beliefs and changes in the wealth distribution}

Theorem 3 shows that, without restrictions on beliefs, the described close link between stock return volatility and interest rate volatility can be broken in our OLG model. Put differently, the theorem suggests that the OLG model, via movements in the wealth distribution, can simultaneously generate substantial stock price volatility and modest interest rate volatility. In this section we investigate the influence of different specifications of heterogeneous beliefs on the wealth distribution as well as on asset prices.

Agents' endowments are given by the afore-mentioned estimated life-cycle income process. Both endowments and dividends exhibit no uncertainty. If beliefs are identical in such an economy, then the unique long-run equilibrium is a steady state with a constant wealth distribution and constant asset prices. For economies with heterogeneous beliefs, however, the predictions of the model are dramatically different. We consider three different specifications of beliefs.

\subsection{Specification of beliefs}

There are three types of agents in the OLG economy. A common feature of all three specifications is that agents of type 1 always hold the correct beliefs. In the first specification, termed "persistent subjective beliefs", agents of types 2 and 3 have beliefs deviating antisymmetrically from the correct beliefs. Beliefs of agents of the same type are identical across generations. We vary both the proportion of type 1 agents as well as the magnitude of belief differences for agents of the other two types. This specification allows us to clearly understand the role of belief heterogeneity for the intergenerational wealth distribution and asset prices. A perhaps unattractive feature of this 
beliefs specification is the lack of learning on behalf of the agents. A possible interpretation (and justification) of this set-up is that the agents receive signals and disagree on their interpretation (see e.g. Acemoglu et al., 2006, or Xiouros, 2010). It is beyond the scope of this paper to introduce a coherent theory of belief heterogeneity. We simply take some specifications as given and explore their implications.

While we need to remain silent on learning, introducing Bayesian learning in our framework renders the model intractable, we examine two modifications of the first specification. In the second beliefs specification, termed "converging beliefs", agents' beliefs converge to the correct beliefs as they age. That is, agents of types 2 and 3 always enter the economy with incorrect prior beliefs but 'learn' while they are alive. In the third specification, termed "temporary disagreement", agents of types 2 and 3 typically have the correct beliefs but with low probability a regime shift occurs. After such a shift, type 2 and 3 agents have temporarily antisymmetric incorrect beliefs. With identical and correct probability, all agents believe that the economy returns to the agreement state. This specification has the advantage that we can view the disagreement states as a structural break in the sense of Cogley and Sargent (2008). Changes in belief heterogeneity over time have been empirically well documented and have important implications for option prices (see e.g. Buraschi and Jiltsov, 2006).

\subsection{Persistent subjective beliefs}

Throughout this first specification of the model, we assume that there are $S=2$ i.i.d. and equiprobable shocks, that is, the data-generating Markov chain is given by $\Pi(1,1)=\Pi(1,2)=\Pi(2,1)=$ $\Pi(2,2)=1 / 2$.

Using micro-data, Gourinchas and Parker (2002) estimate the annual discount rate to be around 0.97. This figure corresponds to a quarterly discount factor of 0.9924 . Alternatively, we can choose $\delta$ to match the average real riskless rate (of about 1 percent p.a.). We report the risk-free rate from our specifications below and see that for many specifications we need a value of $\delta$ above 1 to match the interest rate. Thus we vary agents' discount factor and examine values of $\delta$ in $\{0.99,1.0,1.01\}$. For the specification of beliefs, we assume that both agents believe (correctly) that the process is i.i.d. Type 2 agents' beliefs satisfy

$$
\pi^{a, 2}(1,1)=\pi^{a, 2}(2,1)=1 / 2+\varepsilon, \pi^{a, 2}(1,2)=\pi^{a, 2}(2,2)=1 / 2-\varepsilon, \quad a=1, \ldots, N-1
$$

while agents of type 3 assume the following transition probabilities,

$$
\pi^{a, 3}(1,1)=\pi^{a, 3}(2,1)=1 / 2-\varepsilon, \pi^{a, 3}(1,2)=\pi^{a, 3}(2,2)=1 / 2+\varepsilon, \quad a=1, \ldots, N-1 .
$$

We denote the fraction of type 1 agents by $\lambda$. 


\subsubsection{Second moments}

Table 4 reports the volatilities $S t d\left(R^{f}\right)$ and $S t d\left(R^{e}\right)$. All entries are in percent. Unless the fraction

\begin{tabular}{|c|c|cc|cc|cc|cc|}
\hline$\delta$ & $\lambda$ & \multicolumn{2}{|c|}{$\varepsilon=0.1$} & \multicolumn{2}{|c|}{$\varepsilon=0.2$} & \multicolumn{2}{c|}{$\varepsilon=0.3$} & \multicolumn{2}{c|}{$\varepsilon=0.4$} \\
\hline 0.99 & 0 & 0.58 & 3.07 & 0.71 & 4.60 & 0.35 & 3.93 & 0.10 & 2.61 \\
\hline 0.99 & $10^{-3}$ & 0.57 & 3.04 & 2.47 & 8.84 & 3.03 & 9.90 & 3.53 & 8.98 \\
\hline 0.99 & 0.1 & 0.47 & 2.30 & 1.01 & 5.04 & 1.11 & 5.58 & 1.37 & 5.59 \\
\hline 0.99 & 0.3 & 0.35 & 1.73 & 0.55 & 3.39 & 0.64 & 3.87 & 0.65 & 4.04 \\
\hline 0.99 & 0.5 & 0.26 & 1.33 & 0.34 & 2.41 & 0.37 & 2.83 & 0.41 & 2.95 \\
\hline 0.99 & 0.9 & 0.01 & 0.55 & 0.01 & 0.82 & 0.01 & 0.95 & 0.13 & 0.98 \\
\hline \hline 1 & 0 & 0.67 & 5.26 & 0.77 & 8.59 & 0.48 & 8.81 & 0.11 & 6.78 \\
\hline 1 & $10^{-3}$ & 0.67 & 5.22 & 2.69 & 12.81 & 3.27 & 14.51 & 3.83 & 13.04 \\
\hline 1 & 0.1 & 0.56 & 3.89 & 1.15 & 8.14 & 1.29 & 9.18 & 1.59 & 9.12 \\
\hline 1 & 0.3 & 0.41 & 2.88 & 0.65 & 5.73 & 0.76 & 6.71 & 0.78 & 7.00 \\
\hline 1 & 0.5 & 0.31 & 2.20 & 0.41 & 4.17 & 0.45 & 5.05 & 0.50 & 5.27 \\
\hline 1 & 0.9 & 0.01 & 0.89 & 0.02 & 1.41 & 0.01 & 1.74 & 0.01 & 1.82 \\
\hline \hline 1.01 & 0 & 1.13 & 8.69 & 1.48 & 16.08 & 1.01 & 17.62 & 0.18 & 14.31 \\
\hline 1.01 & $10^{-3}$ & 1.29 & 8.37 & 3.14 & 17.26 & 3.50 & 18.46 & 3.81 & 16.50 \\
\hline 1.01 & 0.1 & 0.88 & 6.11 & 1.39 & 11.27 & 1.53 & 12.28 & 1.86 & 11.98 \\
\hline 1.01 & 0.3 & 0.61 & 4.40 & 0.83 & 8.17 & 0.94 & 9.34 & 0.97 & 9.47 \\
\hline 1.01 & 0.5 & 0.46 & 3.33 & 0.55 & 6.11 & 0.59 & 7.20 & 0.67 & 7.34 \\
\hline 1.01 & 0.9 & 0.19 & 1.29 & 0.22 & 2.18 & 0.18 & 2.60 & 0.21 & 2.76 \\
\hline
\end{tabular}

Table 4: Volatility $\left(\operatorname{Std}\left(R^{f}\right)\right.$ and $\operatorname{Std}\left(R^{e}\right)$ in $\left.\%\right)$ - persistent differences in beliefs

of agents with correct beliefs is large (50 or 90 percent) and/or discounting is relatively low ( $\delta=$ 0.99), the stock return volatility exceeds the corresponding value for the OLG economy with the largest dividend uncertainty and identical beliefs, see Table 2 in the previous section. The stock return volatility increases with $\delta$. It is non-monotone in the belief parameter $\varepsilon$ and the fraction $\lambda$ of agents with correct beliefs.

For $\lambda=0$ the stock return volatility increases substantially from $\varepsilon=0.1$ to $\varepsilon=0.2$ but then levels off or even decreases as $\varepsilon$ increases further. The economic reason for the declining stock return (and interest rate) volatility is straightforward. For large values of $\varepsilon$, the relatively old agents in the economy become poor with very high probability and are thus at their natural borrowing constraint. They have no or little wealth left to invest and so their share of the wealth distribution remains small over time. The declining movements in the wealth distribution lead to declining asset price volatility. 
The dependence of the stock return and interest rate volatilities on the belief parameter $\varepsilon$ is markedly different when a very small fraction of agents has the correct beliefs, see the results for $\lambda=10^{-3}$ in Table 4 . For large values of $\varepsilon$ there is a good chance that the old type 1 agents retire quite rich. This phenomenon is perfectly in line with the well-known results for infinite-horizon economies. In such economies the small fraction of type 1 agents would eventually hold the entire wealth in the economy. Due to agents' finite life span in the OLG economy, this limit effect does not occur here. Nevertheless the presence of a small fraction of agents with correct beliefs leads to large movements in the wealth distribution and large asset price volatility. The volatility of both the stock return and the interest rate become large as $\varepsilon$ increases. As it becomes more and more likely that the very old hold substantial wealth in the economy, the interest rate volatility can no longer be disentangled from the stock return volatility. For $\lambda=0.9$ both volatilities remain small.

Finally note that in this simple setup without uncertainty, we are able to roughly match observed volatility of stock returns and the real interest rate. In Section 3 we explained why this is always theoretically possible without any restrictions on beliefs. Interestingly, heterogenous beliefs within a generation that are constant across generations create enough movements in the intergenerational wealth distribution to generate similar effects. We interpret these results further once we describe the movements in the wealth distribution below. First we turn to the first moments.

\subsubsection{The risk-free rate and the market price of risk}

Not surprisingly, our parsimonious OLG model fails to simultaneously match observed risk-free interest rates and stock returns. Markets are dynamically complete and agents' risk aversion is too low. Nevertheless we discuss the first moments because they shed some light on the economic forces present in the model. Table 5 reports the average (quarterly) interest rate and the equity premium (both in percent) for economies with a discount factor of $\delta=1$.

The equity premium is positive only for $\lambda=0$. In such OLG economies, most of the time younger agents hold all the wealth. But the stock is not really an attractive asset for them because it loses value just as the agents get poorer. Thus, the equity premium must be positive for the agents to buy the stock. As soon as a small fraction of agents has correct beliefs, the equity premium drops substantially and even becomes negative. The agents with correct beliefs are (likely to be) rich in old age and then hold most of the wealth in the economy. For them the stock is the only investment vehicle to save for retirement and so they do not demand high excess returns. As the fraction $\lambda$ of (type 1) agents with correct beliefs increases and tends to one, the equity premium becomes less negative and approaches zero. If all agents had the correct beliefs, that is, in the absence of "belief risk" in the stock, the stock would be risk-free and redundant even though it is an infinitely-lived security and the risk-free bond is a one-period asset. So, for $\lambda=1$ the equity premium would be zero. 


\begin{tabular}{|c|c|r|r|r|r|}
\hline$\lambda$ & Moment & $\varepsilon=0.1$ & $\varepsilon=0.2$ & $\varepsilon=0.3$ & $\varepsilon=0.4$ \\
\hline 0 & $E\left(R^{f}\right)$ & 0.25 & 0.00 & -0.01 & -0.01 \\
\hline 0 & $E\left(R^{e}-R^{f}\right)$ & 0.37 & 0.90 & 1.04 & 1.00 \\
\hline 0.1 & $E\left(R^{f}\right)$ & 0.54 & 1.75 & 2.20 & 2.33 \\
\hline 0.1 & $E\left(R^{e}-R^{f}\right)$ & 0.09 & -0.32 & -0.28 & -0.23 \\
\hline 0.3 & $E\left(R^{f}\right)$ & 0.60 & 1.21 & 1.44 & 1.54 \\
\hline 0.3 & $E\left(R^{e}-R^{f}\right)$ & 0.01 & -0.21 & -0.21 & -0.19 \\
\hline 0.5 & $E\left(R^{f}\right)$ & 0.59 & 0.93 & 1.01 & 1.01 \\
\hline 0.5 & $E\left(R^{e}-R^{f}\right)$ & 0.01 & -0.13 & -0.14 & -0.14 \\
\hline 0.9 & $E\left(R^{f}\right)$ & 0.57 & 0.61 & 0.66 & 0.67 \\
\hline 0.9 & $E\left(R^{e}-R^{f}\right)$ & 0.01 & -0.01 & -0.02 & -0.03 \\
\hline
\end{tabular}

Table 5: First moments (in \%) - persistent differences in beliefs

\subsubsection{The wealth distribution}

Recall that in the benchmark economy in Section 3.1 the wealth distribution has a dramatic impact on asset prices while in the calibrated example with aggregate uncertainty this effect is absent simply because the wealth distribution does not move. Theorem 3 shows that with arbitrary heterogeneous beliefs substantial movements in the wealth distribution are possible. We now document that large effects are present in the calibrated economy.

We report results for the case $\delta=1, \lambda=0.3$, and $\varepsilon=0.2$. Table 6 displays the average cashat-hand shares of all ten groups as well as the corresponding standard deviations and also the correlation coefficient of the cash-at-hand shares and the stock price.

\begin{tabular}{|l|rrrrr|}
\hline Group & 1 & 2 & 3 & 4 & 5 \\
\hline average (\%) & 1.17 & -5.55 & -8.47 & -5.54 & 2.24 \\
\hline std. dev. (\%) & 18.29 & 26.21 & 22.10 & 17.26 & 15.49 \\
\hline$r(p)$ & 0.3579 & 0.6228 & 0.5554 & 0.3241 & 0.0458 \\
\hline \hline Group & 6 & 7 & 8 & 9 & 10 \\
\hline average (\%) & 12.99 & 24.99 & 33.01 & 30.30 & 14.94 \\
\hline std. dev. (\%) & 15.34 & 16.53 & 17.63 & 15.84 & 8.21 \\
\hline$r(p)$ & -0.2519 & -0.5050 & -0.6633 & -0.7341 & -0.7203 \\
\hline
\end{tabular}

Table 6: Wealth distribution - persistent differences in beliefs

As we would expect in an OLG model, the second five groups (of older agents) hold, on average, a much larger share of aggregate wealth than the first five (younger) groups. Cash-at-hand 
shares peak for group 8 (agents living in their 169th to 192nd period, retiring in period 173) and decrease subsequently. The shares do not grow monotonically across the age groups but fall until group 3. In fact, groups 2, 3, and 4 have negative average shares. This effect is a result of the agents with wrong beliefs losing (on average) money on their investments.

The standard deviations of the cash-at-hand shares range from $8 \%$ to more than $26 \%$. These figures indicate that the wealth distribution in this economy is very volatile. Groups 1, 2, and 3 have the largest standard deviation of cash-at-hand shares, so agents experience the largest volatility during the earlier periods of their lives. Since the agents are unconstrained and have their most productive periods with the highest labor income ahead of them, they can make the largest "bets" on the states they deem most likely. These investments result in volatile wealth levels for them depending on the realization of the exogenous states.

The correlation coefficients highlight the impact of the wealth distribution on the stock price. The cash-at-hand shares of the first five groups are positively correlated with the stock price; the shares of the second five groups are negatively correlated with the stock price. The coefficients for groups 2 and 3 have the highest positive value, those for groups 9 and 10 the most negative value. (All ten coefficients are significant.) Figures 1 and 2 visualize the correlation between the stock price and the wealth shares of groups 2 and 9, respectively. Figure 1 shows the positive correlation between the wealth share of group 2 and the stock price. Figure 2 shows the negative correlation between the wealth share of group 9 and the stock price. Simply put, when the young are rich, the stock price tends to be high; when the old are rich, the stock price tends to be low. The results in Table 6 and the two graphs clearly document a main theme of this paper, namely that movements in the wealth distribution lead to changes in asset prices.

\section{[FIGURES 1 AND 2 ABOUT HERE]}

The pricing results above indicate that the fraction of agents with correct beliefs, $\lambda$, has an important effect on the intergenerational wealth distribution. The following table shows the moments of the wealth distribution for the case $\delta=1, \lambda=0.3$, and $\varepsilon=0.2$.

\begin{tabular}{|l|rrrrr|}
\hline Group & 1 & 2 & 3 & 4 & 5 \\
\hline average (\%) & 28.33 & 31.10 & 18.46 & 9.45 & 4.37 \\
\hline std. dev. (\%) & 46.97 & 56.55 & 51.29 & 45.18 & 38.54 \\
\hline \hline Group & 6 & 7 & 8 & 9 & 10 \\
\hline average (\%) & 2.43 & 2.38 & 2.27 & 0.97 & 0.24 \\
\hline std. dev. (\%) & 32.28 & 26.66 & 20.49 & 13.41 & 5.45 \\
\hline
\end{tabular}

Table 7: Moments of wealth distribution, $\lambda=0$ - persistent differences in beliefs 
The numbers are in stark contrast to the ones in Table 6 . When $\lambda=0$, the old are very poor, the young very rich and the standard deviations are much higher than in the economy with $\lambda=0.3$. The disparity between the two economies is also reflected in the first moments: for $\lambda=0$, the average interest rate is zero, for $\lambda=0.3$ it exceeds 1 percent.

\subsection{Converging beliefs}

An unappealing feature of our analysis of heterogenous beliefs until now has been their persistence. The agents do not learn and so do not adjust their subjective beliefs as a result of observed relative frequencies of the shocks in the economy. In order to address this issue, we now modify the specification of the economy and assume that the beliefs of the type 2 and type 3 agents within a cohort converge to the correct beliefs. We consider two different speeds of convergence which we term 'slow' and 'fast'. In the first scenario of slow convergence, beliefs converge linearly over time until all three agents in a cohort have the identical and correct beliefs in the very last period. Formally, the beliefs of type 2 and type 3 agents in period $a$ of their lives are

$$
\pi^{a, 2}(1,1)=\pi^{a, 2}(2,1)=\left(\frac{1}{2}+\varepsilon\right)\left(1-\frac{a}{240}\right)+\frac{1}{2} \frac{a}{240},
$$

and

$$
\pi^{a, 3}(1,1)=\pi^{a, 3}(2,1)=\left(\frac{1}{2}-\varepsilon\right)\left(1-\frac{a}{240}\right)+\frac{1}{2} \frac{a}{240},
$$

respectively. In the second scenario of fast convergence, beliefs converge again linearly and are identical after 120 periods. That is, in the second half of their life all three agents in a cohort have identical and correct beliefs.

We report simulation results for economies with $\delta=1$ and $\lambda=0.3$. Table 8 reports the second moments of bond and stock returns for the two convergence scenarios.

\begin{tabular}{|c|cc|cc|cc|cc|}
\hline Case & \multicolumn{2}{|c|}{$\varepsilon=0.1$} & \multicolumn{2}{c|}{$\varepsilon=0.2$} & \multicolumn{2}{c|}{$\varepsilon=0.3$} & \multicolumn{2}{c|}{$\varepsilon=0.4$} \\
\hline Slow & 0.45 & 2.28 & 0.74 & 5.16 & 0.70 & 6.51 & 0.78 & 6.89 \\
\hline Fast & 0.35 & 1.70 & 0.91 & 4.39 & 1.02 & 6.13 & 0.89 & 6.79 \\
\hline
\end{tabular}

Table 8: Volatility $\left(\operatorname{Std}\left(R^{f}\right)\right.$ and $\operatorname{Std}\left(R^{e}\right)$ in \%) - converging beliefs

Comparing the figures in the table to the corresponding row in Table 4, we notice that converging beliefs generate similar quantitative implications as persistent heterogenous beliefs. As discussed previously, agents who hold incorrect beliefs often become relatively rich when they are young but rarely manage to maintain their wealth for a long time. The wealth shares of the groups of young agents fluctuate. The lack of substantial (if any) disagreement between the older agents has only a small impact on the volatility of asset returns.

Table 9 provides information on the wealth distribution for the economy with fast converging beliefs for $\lambda=0.3$ and $\varepsilon=0.2$. In comparison to the economy with persistent subjective beliefs, 
the volatility in the wealth shares for younger agents (groups 1 and 2) slightly decreases while it increases for all other groups. When a cohort with wrong beliefs is lucky in young age and accumulates a lot of wealth, then, with fast converging beliefs, they are more likely to keep this wealth throughout their lifetime. As a result the volatility of their wealth shares increases. In the economy with persistent differences, the wealth share of the old is always determined by the agents with correct beliefs (type 1) since agents of the other types eventually become poor. Therefore, the wealth shares of the older groups vary less.

\begin{tabular}{|l|rrrrr|}
\hline Group & 1 & 2 & 3 & 4 & 5 \\
\hline average (\%) & -0.15 & -4.74 & -5.26 & -1.20 & 6.32 \\
\hline std. dev. (\%) & 15.14 & 23.98 & 23.82 & 22.97 & 23.32 \\
\hline$r(p)$ & 0.2412 & 0.4787 & 0.4685 & 0.3333 & 0.1486 \\
\hline \hline Group & 6 & 7 & 8 & 9 & 10 \\
\hline average (\%) & 15.41 & 24.33 & 29.16 & 24.71 & 11.42 \\
\hline std. dev. (\%) & 23.69 & 23.65 & 23.04 & 19.34 & 9.71 \\
\hline$r(p)$ & -0.0587 & -0.2842 & -0.4886 & -0.6163 & -0.6300 \\
\hline
\end{tabular}

Table 9: Wealth distribution - fast converging beliefs

In sum, the described volatility of the wealth distribution and the resulting asset return volatility are robust to the stylized form of "linear" learning. We would expect to obtain similar results if agents experienced Bayesian learning during their lifetimes. For the robustness of our results it is only important that agents with heterogenous beliefs continually enter the economy. So, the agents do not use past data to form (identical) prior beliefs at their date of birth.

\subsection{Temporary disagreements}

We consider a third specification of heterogenous beliefs in the OLG economy in which all agents agree most of the time but in which temporary 'regime switches'lead to disagreement. We assume that there are three shocks $s=1,2,3$. The true law of motion is as follows,

$$
\Pi=\left[\begin{array}{ccc}
\mathfrak{p} & 1-\mathfrak{p} / 2 & 1-\mathfrak{p} / 2 \\
0.2 & 0.4 & 0.4 \\
0.2 & 0.4 & 0.4
\end{array}\right]
$$

In shock 1, all three types of agents agree and know the true probabilities. As before, agents of type 1 have correct beliefs in all states throughout their life, i.e. $\pi^{a, 1}=\Pi$ for all $a=1, \ldots, N-1$. In shocks 2 and 3 , the beliefs of types 2 and 3 are $a=1, \ldots, N-1$,

$$
\pi^{a, h}(2,1)=\pi^{a, h}(3,1)=0.2, \quad h=2,3
$$


and

$$
\pi^{a, 2}(2,2)=\pi^{a, 2}(3,2)=0.4+\varepsilon \quad \text { and } \quad \pi^{a, 3}(2,2)=\pi^{a, 3}(3,2)=0.4-\varepsilon,
$$

respectively. For an interpretation of the results it is useful to consider the stationary distribution $\left(\hat{\pi}_{1}, \hat{\pi}_{2}, \hat{\pi}_{3}\right)$ of the Markov chain describing the regime shifts in agents' beliefs. Table 10 reports the stationary distribution for three different values of the probability $\Pi(1,1)=\mathfrak{p}$. For $\mathfrak{p}=0.8$ the

\begin{tabular}{|c|c|c|c|}
\hline $\mathfrak{p}$ & $\hat{\pi}_{1}$ & $\hat{\pi}_{2}$ & $\hat{\pi}_{3}$ \\
\hline 0.8 & $\frac{1}{2}$ & $\frac{1}{4}$ & $\frac{1}{4}$ \\
\hline 0.9 & $\frac{2}{3}$ & $\frac{1}{6}$ & $\frac{1}{6}$ \\
\hline 0.99 & $\frac{20}{21}$ & $\frac{1}{42}$ & $\frac{1}{42}$ \\
\hline
\end{tabular}

Table 10: Stationary distribution - temporary disagreement

agents disagree on average 50 percent of the time, for $\mathfrak{p}=0.9$ they disagree $1 / 3$ of the time, and finally for $\mathfrak{p}=0.99$ they disagree once every 21 quarters on average.

Table 11 reports the long-run measures $\operatorname{Std}\left(R^{f}\right)$ and $\operatorname{Std}\left(R^{e}\right)$ for different values of $\varepsilon$ and $\mathfrak{p}$ for an economy with a discount factor of $\delta=1$ and a fraction of type 1 agents of $\lambda=0.3$. Stock

\begin{tabular}{|l|ll|ll|ll|}
\hline $\mathfrak{p} \backslash \varepsilon$ & \multicolumn{2}{|c|}{0.1} & \multicolumn{2}{c|}{0.2} & \multicolumn{2}{c|}{0.3} \\
\hline 0.8 & 0.36 & 2.46 & 0.61 & 4.81 & 0.72 & 6.22 \\
\hline 0.85 & 0.34 & 2.33 & 0.57 & 4.47 & 0.72 & 6.09 \\
\hline 0.9 & 0.31 & 2.07 & 0.54 & 3.94 & 0.70 & 5.63 \\
\hline 0.95 & 0.24 & 1.59 & 0.42 & 3.03 & 0.63 & 4.53 \\
\hline 0.99 & 0.08 & 0.51 & 0.19 & 1.22 & 0.33 & 1.98 \\
\hline
\end{tabular}

Table 11: Volatility $\left(\operatorname{Std}\left(R^{f}\right)\right.$ and $\operatorname{Std}\left(R^{e}\right)$ in \%) - temporary disagreement

returns exhibit considerable volatility even though disagreement is temporary and fundamentals are deterministic. For $\mathfrak{p}=0.8$ and $\epsilon=0.3$ the resulting volatility is close to the one observed in the data. Not surprisingly, as $\mathfrak{p}$ becomes large the return volatility decreases substantially and for $\mathfrak{p}=0.99$ is very small. For $\mathfrak{p}=0.99$ all agents have identical and correct beliefs in more than $95 \%$ of all periods, that is, disagreement is rare. However, for the case $\lambda=0$ (i.e., all agents have incorrect beliefs), the volatility in this specification increases considerably. For example, for $\varepsilon=0.3$ we obtain that $\operatorname{Std}\left(R^{f}\right)=0.89$ and $\operatorname{Std}\left(R^{e}\right)=4.24$.

Since the agents agree most of the time anyway, a very small fraction of agents with correct beliefs does not lead to a large change in the second moment, contrary to our benchmark economies, see Table 4. However, this effect of a very small fraction of agents with correct beliefs is present in economies where disagreement is more common. For example, for $\mathfrak{p}=0.9$, when agents agree 
only $2 / 3$ of the time, we obtain that for $\lambda=0$ the standard deviation of stock returns is 9.91 percent and the standard deviation of the interest rate is 1.11 percent (when $\varepsilon=0.3$ ). If we introduce a small fraction of agents with correct beliefs and set $\lambda=10^{-3}$, this standard deviation increases to 12.80 percent for stock returns and 2.81 percent for the interest rate.

In sum, for many parameter values, the OLG economy with temporary disagreement also leads to high stock return volatility. And again, the volatility of the stock return is substantially larger than the interest rate volatility.

\section{Conclusion}

In this paper we have analyzed asset price volatility in an exchange economy with overlapping generations. Our OLG model is both canonical and parsimonious - financial markets are com-

plete and there are no restrictions on asset trades. We have shown that with identical beliefs the asset pricing implications of this model are similar to the implications of a standard Lucas (1978) model with a representative agent. This finding is in line with the previous literature. However, differences in opinion, even when they are small and unrelated to fundamentals in the economy, can lead to completely different asset pricing implications. The interplay of belief heterogeneity and life-cycle investments leads to considerable changes in the wealth distribution which in turn result in substantial asset price volatility. In fact, the model generates realistic second moments of asset returns.

\section{Appendix}

\section{A Proofs}

For the proofs of Theorem 1, Proposition 1 and 2 it is useful to consider the Arrow-Debreu equilibrium of the OLG economy. In finite Arrow-Debreu economies, the assumption of Cobb-Douglas utility implies that the equilibrium is unique and that equilibrium prices are the solution of a linear system of equations. These insights carry over to the Arrow-Debreu equilibrium of our OLG model although the technical details are more complicated. Since it follows from Santos and Woodford (1997) that there cannot be bubbles in our sequential equilibrium, there is a one-to-one correspondence between the Arrow-Debreu equilibria and the sequential equilibria.

We first define the endowments of each agent appearing in the OLG economy at each node of the event tree. Recall that we identify individuals (born into the economy) by the date-event of their birth, $\sigma \in \Sigma$, and their type $h=1, \ldots, H$. For each such agent define his endowment $\omega^{\sigma, h}\left(s^{t}\right)=e^{\sigma, h}\left(s^{t}\right)$ for all nodes $s^{t} \in \Sigma$. Of course, the definition of $e$ implies that an agent's endowment is zero at all nodes at which he is not alive. Recall that we denote individuals who 
are born before $t=0$ by $\left(s^{1-a}, h\right)$ for $a=2, \ldots, N$ and $h=1, \ldots, H$. These agents' endowments include the dividends of the Lucas-tree over the whole event tree and are thus given by $\omega^{s^{1-a}, h}\left(s^{t}\right)=e^{s^{1-a}, h}\left(s^{t}\right)+\phi^{s^{1-a}, h} d\left(s^{t}\right)$ for all nodes $s^{t} \in \Sigma$. The aggregate endowment in the economy is

$$
\omega\left(s^{t}\right)=\omega\left(s_{t}\right)=\sum_{\sigma \in \Sigma} \sum_{h=1}^{H} \omega^{\sigma, h}\left(s^{t}\right)+\sum_{a=2}^{N} \sum_{h=1}^{H} \omega^{-a+1, h}\left(s^{t}\right) .
$$

Note that the aggregate endowment only depends on the current shock $s_{t}$. Denote the price for the consumption good at each node $s^{t} \in \Sigma$ by $\rho\left(s^{t}\right)$ with the normalization $\rho\left(s_{0}\right)=1$.

The Arrow-Debreu equilibrium is defined as usual by prices $(\rho(\sigma))_{\sigma \in \Sigma}$ and consumption allocations $c^{\sigma, h}$ such that markets clear and agents maximize utility.

Observe that a finite number of agents, namely those born before $t=0$, hold a non-negligible fraction of the aggregate endowment at all nodes. This fact ensures the existence of a competitive equilibrium, see Geanakoplos and Polemarchakis (1991, Theorem 2). Moreover, in every equilibrium the value of the aggregate endowment must be finite. Our assumption of log utility implies that excess demand functions satisfy the gross substitute property. As a consequence of these last two properties the Arrow-Debreu equilibrium is unique, see Kehoe et al. (1991, Theorem A).

Building on the existence of a unique Arrow-Debreu equilibrium we can now prove Theorem 1.

Proof of Theorem 1. We first determine the income of all agents in the economy. The income of agent $\left(s^{1-a}, h\right)$ who is born before $t=0$ is given by

$$
I^{s^{1-a}, h}=\phi^{s^{1-a}, h} \sum_{\sigma \in \Sigma} \rho(\sigma) d(\sigma)+\sum_{n=a}^{N} \sum_{s^{n-a} \succeq s_{0}} \rho\left(s^{n-a}\right) e^{n, h}\left(s_{n-a}\right)
$$

for $a=2, \ldots, N$ and $h=1, \ldots, H$. Analogously to our cash-at-hand definition for the sequential equilibrium we can define cash-at-hand for the Arrow-Debreu equilibrium as

$$
\kappa^{s^{1-a}, h}=\phi^{s^{1-a}, h} \sum_{\sigma \in \Sigma} \rho(\sigma) d(\sigma)
$$

for $a=2, \ldots, N-1$ and $h=1, \ldots, H$. With this expression we can rewrite agents' income as follows,

$$
\begin{aligned}
I^{s^{1-a}, h} & =\kappa^{s^{1-a}, h}+\sum_{n=a}^{N} \sum_{s^{n-a} \succeq s_{0}} \rho\left(s^{n-a}\right) e^{n, h}\left(s_{n-a}\right) \quad \text { for } a=2, \ldots, N-1, \\
\sum_{h=1}^{H} I^{s^{1-N}, h} & =\sum_{\sigma \in \Sigma} \rho(\sigma) d(\sigma)-\left(\sum_{a=2}^{N-1} \sum_{h=1}^{H} \kappa^{s^{1-a}, h}\right)+\rho\left(s_{0}\right) \sum_{h=1}^{H} e^{N, h}\left(s_{0}\right) .
\end{aligned}
$$

The income of agent $(\sigma, h)$ entering the economy at node $\sigma=s^{t} \in \Sigma$ is given by

$$
I^{\sigma, h}=\sum_{a=1}^{N} \sum_{s^{t+a-1} \succeq \sigma} \rho\left(s^{t+a-1}\right) e^{a, h}\left(s_{t+a-1}\right) .
$$


Next we derive the linear system of excess demand equations that determines equilibrium prices. This system is analog to the system in finite economies with Cobb-Douglas utilities with the exception that it has infinitely many equations and unknowns. For each agent $s^{t}, h$ and age $a=1, \ldots, N$ define the weight

$$
\xi^{s^{t}, h}\left(s^{t+a-1}\right)=\frac{\delta^{a-1} \pi^{s^{t}, h}\left(s^{t+a-1} \mid s^{t}\right)}{\sum_{j=0}^{N-1} \delta^{j}} .
$$

For the agents $\left(s^{1-a}, h\right)$ who are present before $t=0$ the coefficients $\xi^{s^{1-a}, h}\left(s^{t}\right)$ are given by

$$
\xi^{s^{1-a}, h}\left(s^{t}\right)=\frac{\delta^{t} \pi^{s^{1-a}, h}\left(s^{t} \mid s_{0}\right)}{\sum_{j=0}^{N-a} \delta^{j}}, \quad t=0, \ldots, N-a,
$$

for $a=2, \ldots, N$ and $s^{t}$ with $t=0, \ldots, N-a$. Observe that in the case $a=N$,

$$
\xi^{s^{1-N}, h}\left(s_{0}\right)=1 \text { for all } h=1, \ldots, H
$$

The Arrow-Debreu prices, normalized such that $\rho\left(s_{0}\right)=1$, are now the unique solution to the following linear system of equations.

$$
\begin{aligned}
\sum_{a=1}^{N} \sum_{h=1}^{H} \xi^{s^{1-a}, h}\left(s_{0}\right) I^{s^{1-a}, h} & =\omega\left(s_{0}\right) \\
\sum_{a=1}^{N} \sum_{h=1}^{H} \xi^{s^{t+1-a}, h}\left(s^{t}\right) I^{s^{t+1-a}, h} & =\rho\left(s^{t}\right) \omega\left(s^{t}\right), \quad \text { for all } s^{t} \succ s_{0}
\end{aligned}
$$

Observe that we can eliminate the income variables of the agents who are of age $N$ at $t=0$ from Equation (14) since they both appear with a weight of 1, see Condition (13), by the right-hand side of Equation (10). These two income variables do not appear in Equations (15). Moreover, we can replace the incomes of all other agents by the corresponding expressions from Equations (9) and (11). A close inspection of the resulting system of infinitely many equations and unknowns reveals that all equations are linear in the cash-at-hand positions $\kappa^{s^{1-a}, h}$ for $a=2, \ldots, N-1$, $h=1, \ldots, H$, and the unknown Arrow-Debreu prices $\rho\left(s^{t}\right)$ for $s^{t} \succ s_{0}$. Therefore, the ArrowDebreu prices are a linear (affine) function of the initial positions $\kappa^{s^{1-a}, h}$. As a result the incomes and thus the consumption allocations of all agents are linear functions of the initial conditions. In particular, the individual consumption allocations at $s_{0}$ are linear in the cash-at-hand positions. The same must be true for the price of the Lucas-tree, $q\left(s_{0}\right)=\sum_{s^{t} \succ s_{0}} \rho\left(s^{t}\right) d\left(s^{t}\right)$, and the price of a riskless one-period bond, $1 / R_{f}\left(s_{0}\right)=\sum_{s_{1} \in \mathcal{S}} \rho\left(s_{1}\right)$.

Since the Arrow-Debreu equilibrium is unique for all initial conditions, the sequential equilibrium that implements the Arrow-Debreu outcome must be recursive with the state consisting of the exogenous shock $s \in \mathcal{S}$ and the beginning of period cash-at-hand across agents, see Kubler and Schmedders (2002).

Finally, note that all coefficients in the pricing and consumption functions, $\alpha, \beta, \gamma$, must be non-negative: If one of the coefficients were negative, we could find initial conditions for a modified economy where the agents of age $N$ at $t=0$ have arbitrarily large endowments and would 
obtain negative prices or negative individual consumptions.

Proof of Proposition 1. For a model without uncertainty we can simplify the linear system of equations (14)-(15) that determines the Arrow-Debreu prices. Without uncertainty we can identify an agent by the date of his birth, $t$, and his type, $h$. The weights (12) aggregate to

$$
\hat{\xi}^{t, h}(t+a-1)=\frac{\delta^{a-1}}{\sum_{j=0}^{N-1} \delta^{j}}
$$

for $a=1, \ldots, N$. Similarly, expression (11) reduces to

$$
\hat{I}^{t, h}=\sum_{a=1}^{N} \hat{\rho}(t+a-1) e^{a, h}
$$

where $(\hat{\rho}(t))$ denotes the sequence of Arrow-Debreu prices. For the agents who are present before time $t=0$ the weights are

$$
\hat{\xi}^{1-a, h}(t)=\frac{\delta^{t}}{\sum_{j=0}^{N-a} \delta^{j}}, \quad t=0, \ldots, N-a,
$$

for $a=2, \ldots, N$. These agents' income is

$$
\hat{I}^{1-a, h}=\kappa^{1-a, h}+\sum_{j=a}^{N} \hat{\rho}(j-a) e^{j, h} .
$$

In the economy without uncertainty, the Arrow-Debreu prices are therefore determined by the simplified linear system

$$
\begin{aligned}
\sum_{a=1}^{N} \sum_{h=1}^{H} \hat{\xi}^{1-a, h}(0) I^{1-a, h} & =\omega \\
\sum_{a=1}^{N} \sum_{h=1}^{H} \hat{\xi}^{t+1-a, h}(t) I^{t+1-a, h} & =\hat{\rho}(t) \omega, \quad \text { for all } t=1,2, \ldots
\end{aligned}
$$

where $\omega \equiv \omega(t), t=0,1, \ldots$, denotes the constant aggregate endowment.

In an economy with deterministic fundamentals but several states we have for any $t$ that

$$
\sum_{s^{t} \in \Sigma} \xi^{s^{t-a+1}, h}\left(s^{t}\right) I^{s^{t-a+1}, h}=\hat{\xi}^{t-a+1, h}(t) \hat{I}^{t-a+1, h} .
$$

with $\sum_{s^{t} \in \Sigma} \rho\left(s^{t}\right)=\hat{\rho}(t)$. (Note that we must add over all possible date-events $s^{t}$ in the event tree at time $t$.) It is easy to see that the prices $\rho\left(s^{t}\right)$ solve the general system of linear equations (14) and (15) if and only if the prices $\hat{\rho}(t)$ solve the specialized system (16) and (17). Since the general system has a unique solution so does the specialized system. This solution does not depend on agents' beliefs and thus the same must be true for the price of the risk-free bond and the price of the Lucas-tree. The consumption of an agent of age $a$ and type $h$ alive at $t=0$ is given by $\hat{\xi}^{1-a, h}(0) I^{1-a, h}$. Clearly this is the same linear function of cash-at-hand for all beliefs. 
Proof of Proposition 2. Equations (16) and (17) in the proof of Proposition 1 determine the Arrow-Debreu prices in a deterministic economy. We rewrite Equation (17) for $t=1$ and the special case $e^{a, h}=0$ for $a>1$ and $e^{1,1}=1$. For simplicity we write this equation recursively and denote by $\kappa^{a}$ the cash-at-hand of agents of age $a($ at $t=0)$. We obtain

$$
\frac{1}{\sum_{j=0}^{N-1} \delta^{j}} \hat{\rho}(1)+\frac{\delta}{\sum_{j=0}^{N-1} \delta^{j}}+\frac{\delta}{\sum_{j=0}^{N-2} \delta^{j}} \kappa^{2}+\ldots+\frac{\delta}{1+\delta} \kappa^{N-1}=\hat{\rho}(1)(1+d) .
$$

Note that the income of the agent of age 2 at $t=1$ is $\hat{I}^{0}=\hat{\rho}(0) e^{1}=1$, while the income of the agent of age 1 is $\hat{\rho}(1)$. Solving for the bond price, $\hat{\rho}(1)$, then gives the desired result.

Derivation of Equation (8). Following Equation (6) the price of the riskless bond is

$$
\begin{aligned}
1 / R^{f}\left(s^{t}\right) & =\gamma_{1}+\sum_{a=2}^{N-1} \gamma_{a} \phi^{a-1}\left(s^{t}\right) \frac{\frac{N-1}{N}+d}{1-\sum_{a=2}^{N-1} \beta_{a} \phi^{a-1}\left(s^{t}\right)} \\
& =\frac{1}{N(d+1)-1}+\left(\frac{N-1}{N}+d\right) \frac{\sum_{a=2}^{N-1} \gamma_{a} \phi^{a-1}\left(s^{t}\right)}{1-\sum_{a=2}^{N-1} \beta_{a} \phi^{a-1}\left(s^{t}\right)} \\
& =\frac{1}{N(d+1)-1}+ \\
& \frac{N(d+1)-1}{N}\left(\frac{N}{N(d+1)-1}\right) \frac{\sum_{a=2}^{N-1}\left(\frac{1}{N-a+1}\right) \phi^{a-1}\left(s^{t}\right)}{1-\sum_{a=2}^{N-1}\left(1-\frac{1}{N-a+1}\right) \phi^{a-1}\left(s^{t}\right)} \\
= & \frac{1}{N(d+1)-1}+\frac{\sum_{a=2}^{N-1}\left(\frac{1}{N-a+1}\right) \phi^{a-1}\left(s^{t}\right)}{1-\sum_{a=2}^{N-1}\left(1-\frac{1}{N-a+1}\right) \phi^{a-1}\left(s^{t}\right)}
\end{aligned}
$$

Proof of Theorem 2. We prove the theorem by a "guess and verify" approach. We guess that consumption allocations are collinear and then derive values for all endogenous variables that satisfy the equilibrium equations.

Suppose that consumption only depends on the shock and that individual consumption allocations are given by $c_{s}^{a, h}=\nu_{a, h} \omega_{s}$ with $\nu_{a, h}>0$ and $\sum_{a=1}^{N} \sum_{h=1}^{H} \nu_{a, h}=1$ for all states $s=1,2, \ldots, S$. Substituting these consumption allocations into the Euler equations yields the prices of the Arrow securities,

$$
q_{s s^{\prime}}=\delta \Pi\left(s, s^{\prime}\right) \frac{\nu_{a, h} \omega_{s}}{\nu_{a+1, h} \omega_{s^{\prime}}}, \quad \forall a=1, \ldots, N-1, h=1, \ldots, H .
$$

The asset prices $q_{s s^{\prime}}$ are obviously independent of the agent and thus we can define a new constant $f$ such that

$$
f \equiv \delta \frac{\nu_{a, h}}{\nu_{a+1, h}} \quad \forall a=1, \ldots, N-1, h=1, \ldots, H .
$$

We can write an agent's lifetime budget constraint, if he does not initially own shares of the tree, as follows,

$$
c_{s}^{1, h}-e_{s}^{1, h}+\sum_{s^{\prime}=1}^{S} q_{s s^{\prime}}\left(c_{s^{\prime}}^{2, h}-e_{s^{\prime}}^{2, h}+\sum_{s^{\prime \prime}=1}^{S} q_{s^{\prime} s^{\prime \prime}}\left(c_{s^{\prime \prime}}^{3, h}-e_{s^{\prime \prime}}^{3, h}+\sum_{s^{\prime \prime \prime}=1}^{S} q_{s^{\prime \prime} s^{\prime \prime \prime}}\left(c_{s^{\prime \prime \prime}}^{4, h}-e_{s^{\prime \prime \prime}}^{4, h}+\ldots\right)\right)\right)=0 .
$$


Case 1. Individual labor endowments are collinear, that is, for each agent $(a, h)$, there is a weight $\eta_{a, h}$ such that his endowments are given by $e_{s}^{a, h}=\eta_{a, h} \omega_{s}$. Note that the weights $\eta_{a, h} \geq 0$ do not sum to $1, \sum_{a=1}^{N} \sum_{h=1}^{H} \eta_{a, h}<1$, since the social endowment $\omega_{s}$ includes dividends $d_{s}$.

In this case

$$
c_{s}^{1, h}-e_{s}^{1, h}=\left(\nu_{1, h}-\eta_{1, h}\right) \omega_{s}
$$

$\mathrm{v}$ and using (18) and (19) also

$$
\begin{aligned}
\sum_{s^{\prime}=1}^{S} q_{s s^{\prime}}\left(c_{s^{\prime}}^{2, h}-e_{s^{\prime}}^{2, h}\right) & =\sum_{s^{\prime}=1}^{S} \Pi\left(s, s^{\prime}\right) f \frac{\omega_{s}}{\omega_{s^{\prime}}}\left(\nu_{2, h}-\eta_{2, h}\right) \omega_{s^{\prime}} \\
& =f\left(\nu_{2, h}-\eta_{2, h}\right) \omega_{s} .
\end{aligned}
$$

Similarly,

$$
\sum_{s^{\prime}=1}^{S} q_{s s^{\prime}} \sum_{s^{\prime \prime}=1}^{S} q_{s^{\prime} s^{\prime \prime}}\left(c_{s^{\prime \prime}}^{3, h}-\omega_{s^{\prime \prime}}^{3, h}\right)=f^{2}\left(\nu_{3, h}-\eta_{3, h}\right) \omega_{s}
$$

and so on. Thus, the budget constraint of an agent of type $h$, born at shock $s$ is equivalent to

$$
\left(\sum_{a=1}^{N} f^{a-1}\left(\nu_{a, h}-\eta_{a, h}\right)\right) \omega_{s}=0
$$

which in turn is equivalent to

$$
\sum_{a=1}^{N} f^{a-1}\left(\nu_{a, h}-\eta_{a, h}\right)=0 .
$$

The definition of the ratio $f$ implies that $f \nu_{a+1, h}=\delta \nu_{a, h}$ and thus $f^{a-1} \nu_{a, h}=\nu_{1, h} \delta^{a-1}$ and so we obtain for each agent $h$,

$$
\nu_{1, h} \sum_{a=1}^{N} \delta^{a-1}-\sum_{a=1}^{N} f^{a-1} \eta_{a, h}=0 .
$$

These $H$ equations together with the market-clearing condition

$$
\sum_{a=1}^{N} \sum_{h=1}^{H} \nu_{a, h}=\sum_{h=1}^{H}\left(\nu_{1, h} \sum_{a=1}^{N} \frac{\delta^{a-1}}{f^{a-1}}\right)=1
$$

yields a system of $H+1$ equations in the $H+1$ unknowns $f$ and $\nu_{1, h}, h=1, \ldots, H$. Substituting for $\nu_{1, h}$ we obtain a polynomial equation in the single unknown $f$,

$$
f^{N-1} \sum_{a=1}^{N} \delta^{a-1}-\left(\sum_{a=1}^{N} f^{a-1} \sum_{h} \eta_{a, h}\right)\left(\sum_{a=1}^{N}\left(f^{N-a} \delta^{a-1}\right)\right)=0 .
$$

Observe that the polynomial on the left-hand side is of the form $g(f)=\sum_{k=0}^{2 N-2} r_{k} f^{k}$ with coefficients $r_{k}$ satisfying $r_{k}<0$ for $k \neq N-1$ and $r_{N-1}>0$. The classical Sign Rule of Descartes now implies that equation (21) can have at most two positive solutions. Moreover, $g(0)=r_{0}<0$ and $g(f) \rightarrow-\infty$ as $f \rightarrow \infty$. And since $g(1)>0$ the polynomial $g$ has two distinct positive roots, one less than 1 and a second larger than 1 . 
There are no bubbles in this OLG economy, see Santos and Woodford (1997). Moreover, the stationarity of the prices of the Arrow securities implies that the tree price is also stationary, i.e. $p\left(s^{t}\right)=p_{s_{t}}$. Agents' Euler equations then require

$$
p_{s}=\sum_{s^{\prime}} q_{s s^{\prime}}\left(p_{s^{\prime}}+d_{s^{\prime}}\right) \quad \text { for all } \quad s=1, \ldots, S,
$$

or, equivalently,

$$
P=Q(P+d)
$$

where $P(d)$ denotes the $S$-vector of tree prices (dividends) and $Q$ denotes the $(S \times S)$-matrix of prices of Arrow securities. The matrix $Q$ is the element-wise (Hadamard) product of the rank-one positive matrix $\Omega$ with elements $\Omega_{s s^{\prime}}=\omega_{s} / \omega_{s^{\prime}}$ and the matrix $f \Pi$ with largest eigenvalue $f$. Thus the matrix $Q$ has also largest eigenvalue $f$. But for the solution $f>1$ of (21) Equation (22) does not yield a finite solution for $P$. The equation delivers only for $f<1$ a finite price vector, namely $P=[I-Q]^{-1} d$. Therefore, the solution $f<1$ of Equation (21) yields the unique equilibrium. In this equilibrium consumption allocations are collinear. This completes the proof of Case 1 .

Case 2. Only the individual labor endowments of cohort 1 are assumed to be collinear with the social endowment, $e_{s}^{1, h}=\eta_{1, h} \omega_{s}$. Beliefs are i.i.d. so we can write $\Pi_{s^{\prime}} \equiv \Pi\left(s, s^{\prime}\right)$ for all $s=1,2, \ldots, S$. As before we have for cohort 0 ,

$$
c_{s}^{1, h}-e_{s}^{1, h}=\left(\nu_{1, h}-\eta_{1, h}\right) \omega_{s}
$$

Now using (18) and (19) we obtain

$$
\begin{aligned}
\sum_{s^{\prime}=1}^{S} q_{s s^{\prime}}\left(c_{s^{\prime}}^{2, h}-e_{s^{\prime}}^{2, h}\right) & =\sum_{s^{\prime}=1}^{S} \Pi_{s^{\prime}} f \frac{\omega_{s}}{\omega_{s^{\prime}}}\left(\nu_{2, h} \omega_{s^{\prime}}-e_{s^{\prime}}^{2, h}\right) \\
& =f \omega_{s}\left(\nu_{2, h}-\sum_{s^{\prime}=1}^{S} \Pi_{s^{\prime}} \frac{e_{s^{\prime}}^{2, h}}{\omega_{s^{\prime}}}\right) .
\end{aligned}
$$

Similarly,

$$
\sum_{s^{\prime}=1}^{S} q_{s s^{\prime}} \sum_{s^{\prime \prime}=1}^{S} q_{s^{\prime} s^{\prime \prime}}\left(c_{s^{\prime \prime}}^{3, h}-\omega_{s^{\prime \prime}}^{3, h}\right)=f^{2} \omega_{s}\left(\nu_{3, h}-\sum_{s^{\prime \prime}=1}^{S} \Pi_{s^{\prime \prime}} \frac{e_{s^{\prime \prime}}^{3, h}}{\omega_{s^{\prime \prime}}}\right)
$$

and so on. Thus, the budget constraint is equivalent to

$$
\sum_{a=1}^{N} f^{a-1} \nu_{a, h}-\eta_{1, h}-\sum_{a=2}^{N} f^{a-1}\left(\sum_{s^{(i)}=1}^{S} \Pi_{s^{(i)}} \frac{e_{s^{(i)}}^{a, h}}{\omega_{s^{(i)}}}\right)=0
$$

where $s^{\prime}=s^{(1)}, s^{\prime \prime}=s^{(2)}$, and so on. Following the same steps as in Case 1 leads to the single polynomial equation

$$
f^{N-1} \sum_{i=0}^{N-1} \delta^{i}-\left(\eta_{0}+\sum_{i=1}^{N-1} f^{i}\left(\sum_{s^{(i)}=1}^{S} \Pi_{s^{(i)}} \frac{e_{s^{(i)}}^{i}}{\omega_{s^{(i)}}}\right)\right)\left(\sum_{i=0}^{N-1} f^{n-i} \delta^{i}\right)=0 .
$$


in the single unknown $f$. As in Case 1 the left-hand side of this equation is a polynomial $g(f)$ with two sign changes and $g(0)<0, g(1)>0$ and $g(f) \rightarrow-\infty$ for $f \rightarrow \infty$. Thus there are again two solutions but, again, only the solution $f<1$ leads to a well-defined stock price. This completes the proof of Case 2 .

Proof of Theorem 3. We construct an economy with 3 shocks $s=1,2,3$, that exhibits the desired volatility. It suffices to consider an economy with one type per generation, $H=1$. Let $\delta=1$ and set $e^{1}=1$ and $e^{a}=0$ for $a=2, \ldots, N$. Denote by $\bar{p}$ the (hypothetical) price of the tree if all wealth is held by the agent of generation $a=2$ and denote by $\underline{p}$ the price of the tree if all wealth is held by the agent of generation $N-2$. The discussion in Section 3.1 implies that $\bar{p} \geq(N-2)(1+d)$ and $\underline{p} \leq 2+d$. Thus, $\bar{p}$ grows with $N$ and $\underline{p}$ is uniformly bounded for all $N$.

For the described specification of the OLG model, the proof of Proposition 1 (or, in fact, Huffman, 1987) implies that the consumption function of an agent of age $a$ is independent of beliefs and just depends on his cash-at-hand. For $\delta=1$ this function is given by

$$
c^{a}\left(\kappa^{a}\right)=\frac{1}{N-a+1} \kappa^{a} \text { for } a=2, \ldots, N .
$$

Agents of age 1 always consume $1 / N$ and the aggregate consumption of all other agents is $\frac{N-1}{N}+$ $d$. For $a \geq 2$ the consumption function is injective, that is, it is a one-to-one mapping between individuals' cash-at-hand and consumption allocation. Thus there exist $\varepsilon>0$ such the equilibrium tree price exceeds $p^{h}=\bar{p}-0.1$ if and only if $c^{2} \geq(1-\varepsilon)\left(\frac{N-1}{N}+d\right)$ and this price is below $p^{l}=\underline{p}+0.1$ if and only if $c^{N-1} \geq(1-\varepsilon)\left(\frac{N-1}{N}+d\right)$. We now construct an economy for which the equilibrium allocations satisfy these properties and choose the "true" probabilities so that the desired volatility is exhibited in equilibrium.

Choose the true law of motion to be $\Pi(s, 1)=\Pi(s, 2)=1 / 2$ and thus $\Pi(s, 3)=0$ for all $s=1,2,3$. All agents' subjective beliefs are i.i.d., that is, we can write $\pi_{s}^{a}$ to denote the subjective probability that the agent of age $a=1, \ldots, N-1$ attaches to shock $s$ in the next period. Choose $\pi_{1}^{1}<1$ close to one and define the price of the Arrow security for state 1 in the next period by

$$
q_{1}=\pi_{1}^{1} \frac{1}{N\left(\frac{N-1}{N}+d\right)(1-\varepsilon)} .
$$

This is the supporting price that ensures that in the next period the agent of age 2 consumes exactly $\left(\frac{N-1}{N}+d\right)(1-\varepsilon)$ if shock 1 occurs. Whenever the equilibrium price for this Arrow security is below this supporting price, the agent consumes more. Analogously, choose $\pi_{2}^{N-2}<1$ close to one and define for some lower bound on consumption, $\underline{c}$,

$$
q_{2}(\underline{c})=\pi_{2}^{N-2} \underline{c} \frac{1}{\left(\frac{N-1}{N}+d\right)(1-\varepsilon)} .
$$

To achieve market clearing we now have to show that there exist probabilities such that for these prices all other agents choose consumption below $\left(\frac{N-1}{N}+d\right) \varepsilon /(N-2)$ and that consumption of 
agents of age $N-2$ is bounded below by $\underline{c}>0$. Define $\pi_{1}^{a}=\pi_{2}^{a}=\zeta$ for all $a=2, \ldots, N-3$ as well as $\pi_{2}^{1}=\pi_{1}^{N-2}=\zeta$. Clearly we can choose $\zeta \leq \varepsilon$ small enough to ensure that the agent of age 3 consumes below $\left(\frac{N-1}{N}+d\right) \varepsilon /(N-2)$ even if the previous state was $s=1$ and he consumed $(1-\varepsilon)\left(\frac{N-1}{N}+d\right)$ in the previous period when he was of age 2 . This choice of beliefs also ensures that all other agents consume below $\left(\frac{N-1}{N}+d\right) \varepsilon /(N-2)$ on the equilibrium path. In fact, it follows from the first order conditions that it suffices to choose

$$
\zeta=\varepsilon /(N-2) \min \left\{q_{1}, q_{2}(\underline{c})\right\}
$$

Iterating on the first order conditions then yields a lower bound $\underline{c}$ on the consumption of agents of age $N-2$, namely

$$
\underline{c} \geq \frac{\varepsilon^{N-3}}{(N-2)^{N-3}} \frac{1}{N} .
$$

In the constructed equilibrium, the price always exceed $p^{h}$ in state 1 and falls below $p^{l}$ in state 2 . Using the true probabilities we can calculate a lower bound on the variance of the stock return,

$$
\left(p^{h}-p^{l}\right)^{2} \frac{\left(4 d^{2}+4 d\left(p^{h}+p^{l}\right)+3\left(p^{h}\right)^{2}+2 p^{h} p^{l}+3\left(p^{l}\right)^{2}\right)}{16\left(p^{h}\right)^{2}\left(p^{l}\right)^{2}} .
$$

Recall that $p^{l}$ is uniformly bounded above for all $N$ while $p^{h}$ grows linearly in $N$. Thus, the (lower bound on the) standard deviation of the stock return grows beyond any bound as $N$ increases.

Finally, we choose $\pi_{1}^{N-1}=\pi_{2}^{N-1}>0$ sufficiently small to ensure that via equation (8) the bond price never falls much below its maximal value and so the interest rate varies by less than a prespecified $\underline{v}>0$. This completes the proof of the theorem.

\section{B Numerical solution}

Recall that in our OLG economy markets are dynamically complete since each date-event $s^{t}$ has $S$ successor nodes and agents can trade a full set of $S$ Arrow securities at each date-event. Moreover, the equilibrium consumption allocation is unique as the discussion preceding the proof of Theorem 1 in Appendix A demonstrates. But agents' portfolios are not unique in equilibrium since the agents can trade the Lucas-tree in addition to the Arrow securities. Thus, in equilibrium, a continuum of portfolios supports the unique consumption allocation. At each date-event equilibrium portfolios are a one-dimensional subspace of $\mathbb{R}^{S}$. For the computation of the linear policy and pricing functions we exploit this multiplicity of portfolios supporting the equilibrium by imposing an additional restriction. This condition on portfolios then uniquely determines one point in the one-dimensional subspace. 


\section{B.1 Equilibrium equations}

The additional restriction forces the agent of age $N-1$ and type 1 to buy the entire Lucas-tree. He holds it for one period and then sells it in the last, $N$ th, period of his life to the subsequent agent of age $N-1$ and type 1 . All agents of ages $a=1, \ldots, N-2$ and the type $h=2, \ldots, H$ agents of age $N-1$ are only permitted to trade Arrow securities. This choice of equilibrium portfolio greatly simplifies the beginning-of-period cash-at-hand for all agents except for the one of age $N-1$ and type 1 . The respective cash-at-hand positions at date-event $s^{t+1}=\left(s^{t}, s_{t+1}\right)$ are then simply $\kappa^{a+1, h}\left(s^{t+1}\right)=\theta_{s_{t+1}}^{a, h}\left(s^{t}\right)$, that is, an agent's cash-at-hand is just his holding of the Arrow security that pays in the current shock $s_{t+1}$.

With the special choice for the equilibrium portfolios we can now derive a nonlinear system of equations that must hold in equilibrium. Let the current shock be $s \in \mathcal{S}$ and the current cash-athand positions be $\kappa^{a, h}$ for $a=2, \ldots, N-1$, and $h=1, \ldots, H$.

The first set of equations are the necessary and sufficient first-order optimality conditions for the agents' utility maximization problems. The generic first-order conditions with respect to portfolio holdings of the Arrow securities are of the form

$$
-q_{s^{\prime}}\left(s^{t}\right) u^{\prime}\left(c^{a, h}\left(s^{t}\right)\right)+\delta \pi^{a, h}\left(s^{\prime} \mid s\right) u^{\prime}\left(c^{a+1, h}\left(s^{t}, s^{\prime}\right)\right)=0 \quad \text { for } a=1, \ldots, N-1,
$$

where $q_{s^{\prime}}\left(s^{t}\right)$ denotes the price of the Arrow security with a payoff in shock $s^{\prime}$ in the next period. Substituting the expressions (1) and (2) into these first-order conditions yields the following equations.

For $a=1$ :

$$
-q_{s^{\prime}}\left(\alpha_{1 s^{\prime}}^{2, h}+\sum_{j=2}^{N-1} \sum_{i=1}^{H} \alpha_{j i s^{\prime}}^{2, h} \theta_{s^{\prime}}^{j-1, i}\right)+\delta \pi^{1, h}\left(s^{\prime} \mid s\right)\left(e_{s}^{1, h}-\sum_{s^{\prime}} q_{s^{\prime}} \theta_{s^{\prime}}^{1, h}\right)=0 .
$$

For $a=2, \ldots, N-2$ :

$$
-q_{s^{\prime}}\left(\alpha_{1 s^{\prime}}^{a+1, h}+\sum_{j=2}^{N-1} \sum_{i=1}^{H} \alpha_{j i s^{\prime}}^{a+1, h} \theta_{s^{\prime}}^{j-1, i}\right)+\delta \pi^{a, h}\left(s^{\prime} \mid s\right)\left(e_{s}^{a, h}+\kappa^{a, h}-\sum_{s^{\prime}} q_{s^{\prime}} \theta_{s^{\prime}}^{a, h}\right)=0 .
$$

For $a=N-1$ and $h=1$ :

$$
\begin{aligned}
-q_{s^{\prime}} & \left(e_{s^{\prime}}^{N, 1}+\theta_{s^{\prime}}^{N-1,1}+d_{s^{\prime}}+\beta_{1 s^{\prime}}+\sum_{j=2}^{N-1} \sum_{i=1}^{H} \beta_{j i s^{\prime}} \theta_{s^{\prime}}^{j-1, i}\right)+\delta \pi^{N-1,1}\left(s^{\prime} \mid s\right) \\
& \left(e_{s}^{N-1,1}+\kappa^{N-1,1}-\sum_{s^{\prime}} q_{s^{\prime}} \theta_{s^{\prime}}^{N-1,1}-\left(\beta_{1 s}+\sum_{j=2}^{N-1} \sum_{i=1}^{H} \beta_{j i s} \kappa^{j, i}\right)\right)=0 .
\end{aligned}
$$

For $a=N-1$ and $h=2, \ldots, H$ :

$$
-q_{s^{\prime}}\left(e_{s^{\prime}}^{N, h}+\theta_{s^{\prime}}^{N-1, h}\right)+\delta \pi^{N-1, h}\left(s^{\prime} \mid s\right)\left(e_{s}^{N-1, h}+\kappa^{N-1, h}-\sum_{s^{\prime}} q_{s^{\prime}} \theta_{s^{\prime}}^{N-1, h}\right)=0 .
$$


Next we have the first-order condition of the agent of age $N-1$ and type 1 with respect to his holding of the Lucas-tree,

$$
-p\left(s^{t}\right) u^{\prime}\left(c^{N-1,1}\left(s^{t}\right)\right)+\delta \sum_{s^{\prime}=1}^{S} \pi^{N-1,1}\left(s^{\prime} \mid s\right)\left(u^{\prime}\left(c^{N, 1}\left(s^{t}, s^{\prime}\right)\right)\left(d_{s^{\prime}}+p\left(s^{t}, s^{\prime}\right)\right)\right)=0 .
$$

Using the prices of the Arrow securities, see Equation (24), we can write the condition on the price of the Lucas-tree as follows,

$$
-\left(\beta_{1 s}+\sum_{j=2}^{N-1} \sum_{i=1}^{H} \beta_{j i s} \kappa^{j, i}\right)+\sum_{s^{\prime}} q_{s^{\prime}}\left(d_{s^{\prime}}+\beta_{1 s^{\prime}}+\sum_{j=2}^{N-1} \sum_{i=1}^{H} \beta_{j i s^{\prime}} \theta_{s^{\prime}}^{j-1, i}\right)=0 .
$$

This equation completes the set of equations derived from agents' first-order conditions.

We have $S$ market-clearing equations.

$$
\sum_{a=1}^{N-1} \sum_{h=1}^{H} \theta_{s^{\prime}}^{a, h}=0 \quad \text { for } s^{\prime}=1, \ldots, S
$$

The third and last set of equations imposes consistency conditions on the linear consumption functions.

For $a=2, \ldots, N-2, h=1, \ldots, H$, and the agents of age $N-1$ and type $h=2, \ldots, H$ :

$$
\alpha_{1 s}^{a, h}+\sum_{j=2}^{N-1} \sum_{i=1}^{H} \alpha_{j i s}^{a, h} \kappa^{j, i}=e_{s}^{a, h}+\kappa^{a, h}-\sum_{s^{\prime}} q_{s^{\prime}} \theta_{s^{\prime}}^{a, h}
$$

For the agent of age $N-1$ and type 1

$$
\alpha_{1 s}^{N-1,1}+\sum_{j=2}^{N-1} \sum_{i=1}^{H} \alpha_{j i s}^{N-1,1} \kappa^{j, i}=e_{s}^{N-1,1}+\kappa^{N-1,1}-\sum_{s^{\prime}} q_{s^{\prime}} \theta_{s^{\prime}}^{N-1,1}-\left(\beta_{1 s}+\sum_{j=2}^{N-1} \sum_{i=1}^{H} \beta_{j i s} \kappa^{j, i}\right)
$$

Equations (25)-(32) must hold for each $s \in \mathcal{S}$ and each initial condition $\kappa^{a, h}$ for $a=2, \ldots, N-1$ and $h=1, \ldots, H$. For fixed $s$ and fixed initial condition, Equations (25)-(29) consist of $H(N-$ 1) $S+1$ equations. In addition, there are $S$ market-clearing equations. Finally there are $H(N-2) S$ consistency conditions. For all $s \in \mathcal{S}$ combined there are

$$
S(H(N-1) S+1+S)+H(N-2) S
$$

equations. Observe that unlike the first-order conditions and market-clearing equations the consistency conditions appear exactly once and are thus not again multiplied by $S$.

The unknowns in our system of equations are $H(N-2) S$ linear consumption functions (the functions for agents of age 1 do not appear in the equations) with $1+H(N-2)$ coefficients each, 
$S$ price functions for the Lucas-tree with $1+H(N-2)$ coefficients each, $S^{2}$ Arrow security prices and $H(N-1) S^{2}$ portfolio variables for agents' holdings of Arrow securities for all possible combinations of $s$ and $s^{\prime}$.

To determine the coefficients of the linear policy and pricing functions we also need to vary the initial conditions. For each value of $\kappa^{a, h}$ we obtain another set of Equations (25)-(32). We do not increase the number of coefficients but only the number of Arrow security prices and portfolio variables. If we choose $1+H(N-2)$ affinely independent values for the initial conditions then we obtain a system with

$$
\begin{aligned}
& (1+H(N-2))(S(H(N-1) S+1+S)+H(N-2) S) \\
= & (H(N-1)+1)(H(N-2)+1) S^{2}+(H(N-2)+1)^{2} S
\end{aligned}
$$

equations and unknowns. A convenient choice for the initial conditions are the zero vector and all possible unit vectors for $\kappa^{a, h}$ for $a=2, \ldots, N-1$ and $h=1, \ldots, H$. We denote this set of $1+H(N-2)$ values by $\mathcal{G}(\kappa)$.

For interesting model specifications the system of nonlinear equations becomes very large. For example, for $H=2, N=240$ and $S=4$ the system consists of 4,565,844 equations and unknowns. Systems of such size are impossible to solve on a laptop without state-of-the-art software for Newton's method or some other algorithm for nonlinear equations. We solve these systems with a simple but slower iterative method based on a Jacobi scheme.

\section{B.2 Iterative Jacobi method}

At the beginning of an iteration, current iterates are available for the $S(1+H(N-2))$-dimensional coefficient vectors $\alpha^{a, h}$ and $\beta$. For each of the $S(1+H(N-2))$ possible combinations of $s \in \mathcal{S}$ and $\kappa \in \mathcal{G}(\kappa)$ we solve a linear system of equations. Observe that Equations (25)-(28) are linear in $q_{s^{\prime}}$ and $q_{s^{\prime}} \theta_{s^{\prime}}^{a, h}$ for $s^{\prime} \in \mathcal{S}, a=1, \ldots, N-1, h=1, \ldots, H$. We can rewrite the market-clearing equations (30) as

$$
\sum_{a=1}^{N-1} \sum_{h=1}^{H} q_{s^{\prime}} \theta_{s^{\prime}}^{a, h}=0 \quad \text { for } s^{\prime}=1, \ldots, S .
$$

The system (25)-(28) and (33) is a square linear system of $H(N-1) S+S$ equations in the $H(N-$ 1) $S$ unknowns $q_{s^{\prime}} \theta_{s^{\prime}}^{a, h}$ and the $S$ unknowns $q_{s^{\prime}}$. We solve this system with $Q R$ factorization with very small error (close to machine precision).

After we have solved $S(1+H(N-2))$ such systems of linear equations we can determine the new iterate for the coefficient vectors $\alpha^{a, h}$ and $\beta$. Note that after substituting all possible combinations of $s \in \mathcal{S}$ and $\kappa \in \mathcal{G}(\kappa)$ and the just computed accompanying solutions for $q_{s^{\prime}}$ and $q_{s^{\prime}} \theta_{s^{\prime}}^{a, h}$ into Equations (29), (31), and (32) these in turn yield a system of $S(1+H(N-2))$ linear equations in the $S(1+H(N-2))$ unknown new coefficients $\alpha^{a, h}$ and $\beta$. The solution to this linear system replaces the current iterate for the coefficients and serves as the next iterate. Now a new 
iteration starts. This iterative procedure terminates when the infinity norm of two subsequent iterates falls below $10^{-10}$.

\section{B.3 Computation of Aggregate Statistics}

In our model, the transition matrix $\Pi$ determines the probability distribution over exogenous shocks in the next period. For a given shock in the following period the transition function of the endogenous state vector $\kappa$ is deterministic. This mapping is not linear but the cash-at-hand of an agent in the next period can be written as the ratio of two linear functions of cash-at-hand across agents in the current period. This can be seen easily from the analysis above. Both the Arrow-prices $q_{s}$ and the expenditure in Arrow-securities $q_{s} \theta_{s}$ are linear functions of the endogenous state $\kappa$. In our algorithm we therefore not only compute the pricing and consumption-policy coefficients, but we also compute the coefficients of the two functions that determine the transition. We can therefore easily simulate the economy and then compute the moments of interest.

We do not aim to numerically approximate the moments integrated over an invariant distribution. Since the transition function in our economy is not monotone, standard techniques for proving uniqueness of invariant distributions (see e.g. Bhattacharya and Majumdar, 2007, for an overview) cannot be applied in our setting. Moreover, while we can do accuracy analysis for our Monte-Carlo approximations for a given finite horizon $T$, we do not know of any methods to do the same for the invariant distribution.

\section{References}

[1] Acemoglu, D., V. Chernozhukov and M. Yildiz (2006), "Learning and Disagreement in an Uncertain World," discussion paper, MIT.

[2] Benhabib, J., A. Bisin and S. Zhu (2011), "The Distribution of Wealth and Fiscal Policy in Economies with Finitely Lived Agents," Econometrica, 79, 123-157.

[3] Bhattacharya, R. and M. Majumdar (2007), Random Dynamical Systems, Cambridge University Press.

[4] Blume, L. and D. Easley (2006), “If You're so Smart, Why Aren't You Rich? Belief Selection in Complete and Incomplete Markets," Econometrica, 74, 929-966.

[5] Bracha, A. and D.J. Brown (2010), "Affective Decision-Making: A Theory of Optimism-Bias," Cowles Foundation Discussion Paper 1759.

[6] Buraschi, A. and A. Jiltsov (2006), "Model Uncertainty and Option Markets with Heterogeneous Beliefs," The Journal of Finance, 61, 2841-2897. 
[7] Campbell, J.Y. (1999), “Asset Prices, Consumption, and the Business Cycle," in: J.B. Taylor and M. Woodford (eds.), Handbook of Macroeconomics, Vol. I, 1231-1303. Elsevier, Amsterdam.

[8] Citanna A. and P. Siconolfi (2010), "Recursive equilibrium in stochastic OLG economies," Econometrica 78, 309-Ü347.

[9] Cogley, T. and T. Sargent (2008), “The Market Price of Risk and the Equity Premium: A Legacy of the Great Depression?" Journal of Monetary Economics, 55, 454-478.

[10] Davis, S.J., F. Kubler and P. Willen (2006), "Borrowing Costs and the Demand for Equity over the Life Cycle," Review of Economics and Statistics, 88, 348-362.

[11] De Long, J.B., A. Shleifer, L.H. Summers, and R.J. Waldmann (1990), “Noise Trader Risk in Financial Markets," Journal of Political Economy, 98, 703-738.

[12] Geanakoplos, J., M. Magill, and M. Quinzii (2004), "Demography and the Long-Run Predictability of the Stock Market," Brookings Papers on Economic Activities 1:2004, 241-325.

[13] Geanakoplos, J.D. and H.M. Polemarchakis (1991), “Overlapping Generations,” in W. Hildenbrand and H. Sonnenschein (eds.), Handbook of Mathematical Economics, Vol. IV, 1900-1960. Elsevier, Amsterdam.

[14] Gourinchas P.-O. and J.A. Parker (2002), “Consumption over the Life Cycle," Econometrica, $70,47-89$.

[15] Harrison, J.M. and D.M. Kreps (1978), "Speculative Investor Behavior in a Stock Market with Heterogeneous Expectations," Quarterly Journal of Economics, 92, 323-336.

[16] Huffman, G. (1987), “A Dynamic Equilibrium Model of Asset Prices and Transaction Volume," Journal of Political Economy, 95, 138-159.

[17] Judd, K.L., F. Kubler, and K. Schmedders (2003), “Asset Trading Volume with Dynamically Complete Markets and Heterogeneous Agents," Journal of Finance, 58, 2203-2217.

[18] Kehoe, T.J., D.K. Levine, A. Mas-Colell and M. Woodford (1991), “Gross-Substitutability in Large-Square Economies," Journal of Economic Theory, 54, 1-25.

[19] Kogan L., S.A. Ross, J. Wang and M.M. Westerfield (2006), “The Price Impact and Survivial of Irrational Traders," Journal of Finance, 61, 195-229.

[20] Krusell, P. and A. Smith (1998), "Income and Wealth Heterogeneity and the Macroeconomy," Journal of Political Economy, 106, 867-896. 
[21] Kubler, F. and K. Schmedders (2002), "Recursive Equilibria in Economies with Incomplete Markets," Macroeconomic Dynamics, 6, 284-306.

[22] Kurz, M. and M. Motolese (2001), “Endogenous Uncertainty and Market Volatility,” Economic Theory, 17, 497-544.

[23] Lettau, M. and H. Uhlig (2002), “The Sharpe Ratio and Preferences: A Parametric Approach," Macroeconomic Dynamics, 6, 242-265.

[24] Lucas, R.E. (1978), “Asset Prices in an Exchange Economy,” Econometrica, 46, 1429-1445.

[25] Morris, S. (1995), "The Common Prior Assumption in Economic Theory," Economics and Philosophy, 11, 227-253.

[26] Rios-Rull, V. (1996), "Life-Cycle Economies and Aggregate Fluctuations," Review of Economic Studies, 63, 465-490.

[27] Sandroni, A. (2000), “Do Markets Favor Agents Able to Make Accurate Predictions?” Econometrica, 68, 1303-1341.

[28] Santos, M.S. and M. Woodford (1997), "Rational Asset Pricing Bubbles," Econometrica, 65, 19-58.

[29] Scheinkman, J. and W. Xiong (2003), "Overconfidence and Speculative Bubbles," Journal of Political Economy 111, 1183-1220.

[30] Storesletten, K., C. Telmer and A. Yaron (2007), "Asset Pricing with Idiosyncratic Risk and Overlapping Generations," Review of Economic Dynamics, 10, 519-548.

[31] Xiouros, C. (2010), "Differences of Opinion and the Price Volume Relation," available at SSRN: http:/ / ssrn.com/abstract=1365096. 
Figure 1: Wealth share (in\%) of group 2 and stock price

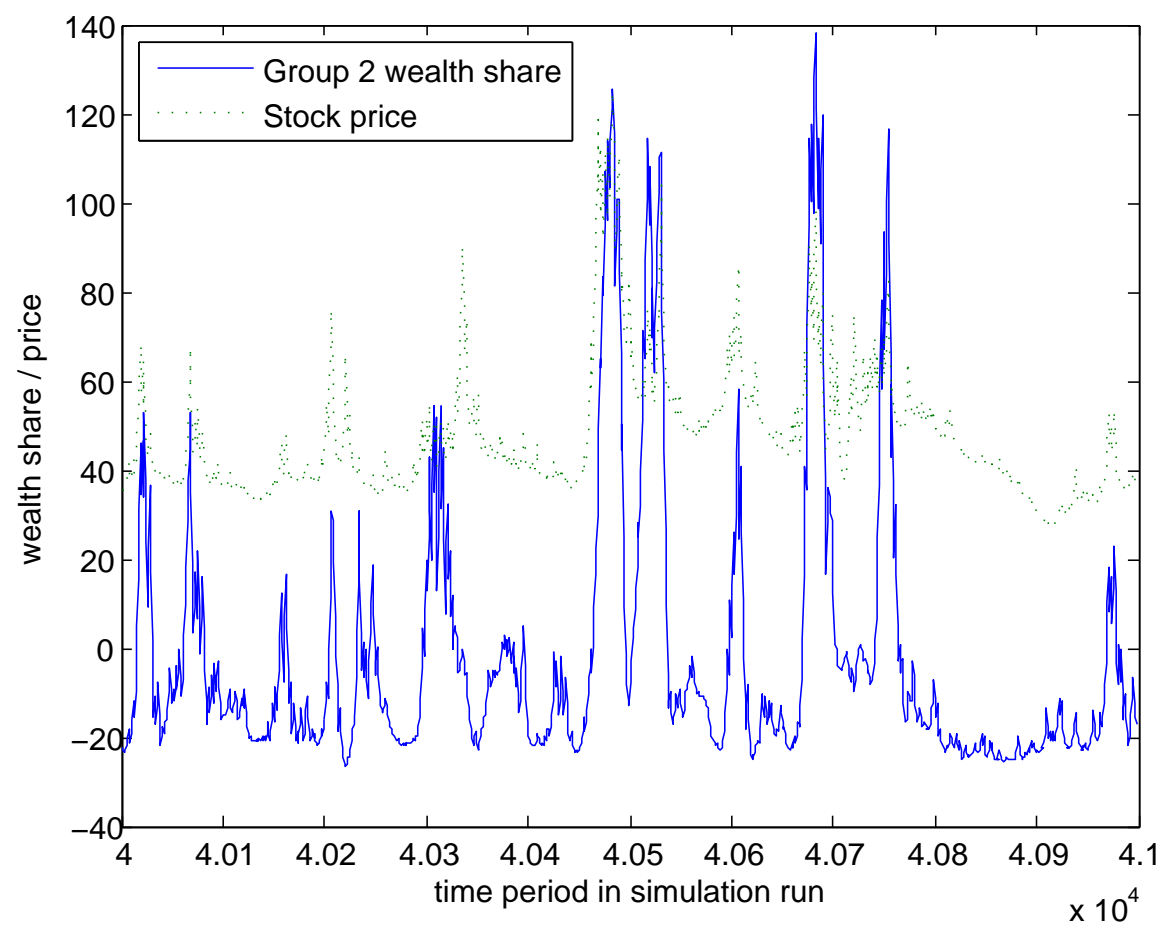

Figure 2: Wealth share (in\%) of group 9 and stock price

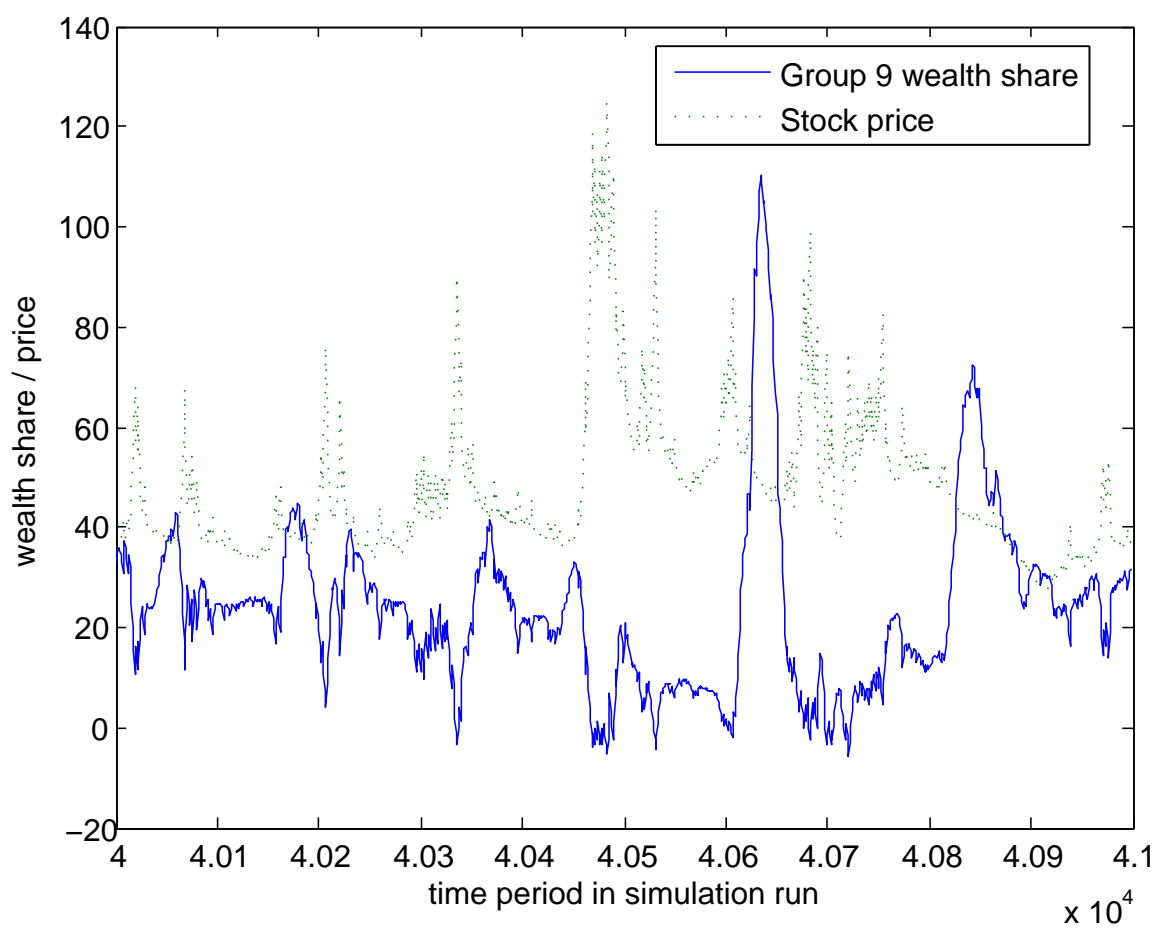

